INRA Prod. Anim., 2011, 24 (1), 89-112

\title{
Impacts et services environnementaux de l'élevage en régions chaudes
}

\author{
V. BLANFORT 1,2,3, M. DOREAU4, J. HUGUENIN,2,3,5, J. LAZARD', V. PORPHYRE 2,3,7, \\ J.-F. SOUSSANA ${ }^{8}$, B. TOUTAIN ${ }^{9}$ \\ ${ }^{1}$ CIRAD, Systèmes d'Elevage Méditerranéens et Tropicaux, BP 701, F- 97387 Kourou, Guyane \\ 2 INRA, UMR0868 Systèmes d'Elevage Méditerranéens et Tropicaux, 2 place Viala, F-34060 Montpellier, France \\ 3 Supagro, Systèmes d'Elevage Méditerranéens et Tropicaux, 2 place Viala, F-34060 Montpellier, France \\ ${ }^{4}$ INRA UR1213 Herbivores, F-63122 Saint-Genès-Champanelle, France \\ ${ }^{5}$ CIRAD, Systèmes d'Elevage Méditerranéens et Tropicaux, Campus International de Baillarguet, F-34398 \\ Montpellier, France \\ ${ }^{6}$ CIRAD, DREI, Avenue Agropolis, F-34398 Montpellier, France \\ ${ }^{7}$ CIRAD, Systèmes d'Elevage Méditerranéens et Tropicaux, 7 chemin de l'IRAT, F-97410 Saint Pierre de la Réunion \\ 8 INRA, UR Ecosystème Prairial, 234 Avenue du Brezet, F- 63100 Clermont-Ferrand, France \\ 9 ex CIRAD UR Pastoralisme, 468 avenue du Miradou, F-34980 Saint Clément de Rivière, France \\ Courriel : blanfort@cirad.fr
}

Les impacts du développement de l'élevage sur l'environnement sont l'objet d'un débat mondial qui prend désormais en compte les services écologiques offerts par les productions animales. L'article propose un bilan et les perspectives de ces interactions élevage/environnement dans les pays du Sud où se réalisera l'essentiel de l'accroissement des productions animales dans les prochaines décennies.

Le débat «élevage/environnement» n'a sans doute jamais été autant abordé qu'en ce début de millénaire. Les productions animales, qui ne contribuent qu'à $1,5 \%$ du PIB mondial selon la FAO (2009), font partie des secteurs les plus impliqués dans les grands enjeux environnementaux aux niveaux local et global. La question est d'autant plus discutée que l'élevage occupe un positionnement complexe en particulier dans les régions chaudes ${ }^{1}$ qui regroupent les zones tropicales, subtropicales et méditerranéennes. Sur les 19 milliards d'animaux d'élevage de la planète, toutes espèces confondues, $70 \%$ appartiennent à des éleveurs hors pays industrialisés ; ils contribuent aux moyens de vie d'un milliard de personnes vulnérables. L'élevage représente $40 \%$ de la Production Agricole Brute mondiale (PAB) et fournit un apport alimentaire en pleine croissance dans les pays en développement (PED) et émergents où s'est réalisé entre 1980 et 2000 l'essentiel des augmentations des productions de viande (triplement), de lait (doublement) et de leur consommation. Les estimations pour 2050 prévoient à nouveau un doublement de ces quantités, essentiellement dans les pays du Sud.

Les zones consacrées à l'élevage d'herbivores comprenant les pâturages spontanés, les prairies et les fourrages semés sont parmi les écosystèmes les plus étendus du monde avec 35 millions de $\mathrm{km}^{2}$, soit 25 à $30 \%$ des terres émergées non gelées. Un tiers des terres cultivées mondiales est consacré à la production d'aliments pour le bétail (Steinfeld et al 2006).

Depuis une vingtaine d'années, un bouleversement de posture s'est engagé, dans lequel les considérations sont passées d'enjeux collatéraux de l'agriculture à des objectifs environnementaux fondamentaux pour l'avenir même de la planète. Il ne s'agit plus simplement de nourrir les hommes mais de considérer l'environnement, la santé, les retombées socio-économiques et politiques. Ces évolutions admises au Nord (sans y être totalement appliquées) sont souvent considérées dans les PED et les pays émergents ${ }^{1}$ comme une vision du «Nord» face aux priorités de lutte contre la faim, la malnutrition, la pauvreté et de développement économique. Les attentes sociétales locales y diffèrent donc des réflexions globales qui nourrissent le débat actuel sur les productions animales et leur évolution.

Ce débat sociétal a été généralement focalisé sur les impacts négatifs de l'élevage sur l'environnement ; il a été largement développé dans le rapport emblématique «Livestock's long Shadow» (l'ombre portée de l'élevage) de Steinfeld et al (2006, version française en 2009). Deux autres documents majeurs ont apporté un point de vue

\footnotetext{
1 Compte tenu de l'objectif de synthèse, nous utiliserons indistinctement dans les parties générales les formulations génériques telles que «pays du Sud», «régions chaudes», «régions tropicales», etc. Au-delà de ce générique climatique, nous utiliserons des unités géographiques plus précises, voire des pays. Une distinction est parfois nécessaire entre «pays en développement» et «pays émergent». Ces derniers ont un PIB par habitant inférieur à celui des pays développés, mais vivent une croissance économique rapide. La liste des pays auxquels s'applique ce terme varie selon les sources et les époques.
} 
nuancé par une plus grande prise en compte des services écologiques offerts par les productions animales (Millennium Ecosystem Assessment 2005, Steinfeld et al 2010). Nous proposons une revue synthétique des impacts et des services écologiques de l'élevage en régions chaudes qui conduit à s'interroger sur la spécificité de la préoccupation environnementale «au Sud», et sur la conception de systèmes d'élevage durables. Quels sont les enjeux de développement et par voie de conséquence de recherche en zone tropicale, au regard des problèmes majeurs de production et d'organisation des élevages et de leurs filières ? Cet article n'est pas une tentative de synthèse exhaustive de l'abondante littérature existant sur le sujet, notamment des volumineux rapports commandités par la FAO. Tout en s'y référant, nous avons surtout tenté d'apporter une vision impliquée d'experts concernés par les préoccupations environnementales actuelles et à venir sur la base de nos expériences dans différents domaines complémentaires des productions animales. Dans une première partie sont retracées les évolutions historiques des interactions de l'élevage et de l'environnement. L'article se structure ensuite autour des enjeux majeurs où l'élevage est fortement impliqué : l'usage des terres et de certains espaces aquatiques, l'utilisation de l'énergie fossile et de l'eau, les émissions de Gaz à Effet de Serre (GES), la biodiversité.

L'élevage est à l'origine de profonds changements des espaces terrestres et de certains espaces aquatiques. Il concerne des surfaces considérables, dépassant celles de l'agriculture proprement dite. Déjà anciens et en quelque sorte plus ou moins stabilisés en régions tempérées, ces changements s'accélèrent dans le monde tropical depuis la fin des années 70 , soit par l'extension et l'évolution rapide des zones vouées à l'élevage, soit par une pression accrue sur les ressources naturelles.

Les répercussions de l'élevage se traduisent également à l'échelle globale de la biosphère à travers les changements climatiques qui sont au cœur des préoccupations environnementales depuis quelques années, occultant d'ailleurs souvent les autres sources d'inquiétude liées aux pollutions, à l'épuisement des ressources et à l'altération des habitats. Les données les plus récentes de l'IPCC (dénomination anglaise du GIEC, Groupe Intergouvernemental d'Experts sur le Climat, IPCC 2007a) font état de scenarii plus préoccupants que ceux proposés il y a seulement quelques années. Les différentes projections s'accordent à prédire une augmentation de température de plusieurs degrés d'ici la fin du siècle.

Photo 1. Départ des troupeaux de zébus Gobra pour le pâturage après l'abreuvement au forage de Tatki dans le Ferlo au Sahel sénégalais. La forte densité quotidienne des animaux autour des forages (plusieurs milliers de têtes) engendre une disparition précoce et localisée de la végétation herbacée en saison sèche dans un rayon d'environ $1 \mathrm{~km}$ autour du forage.

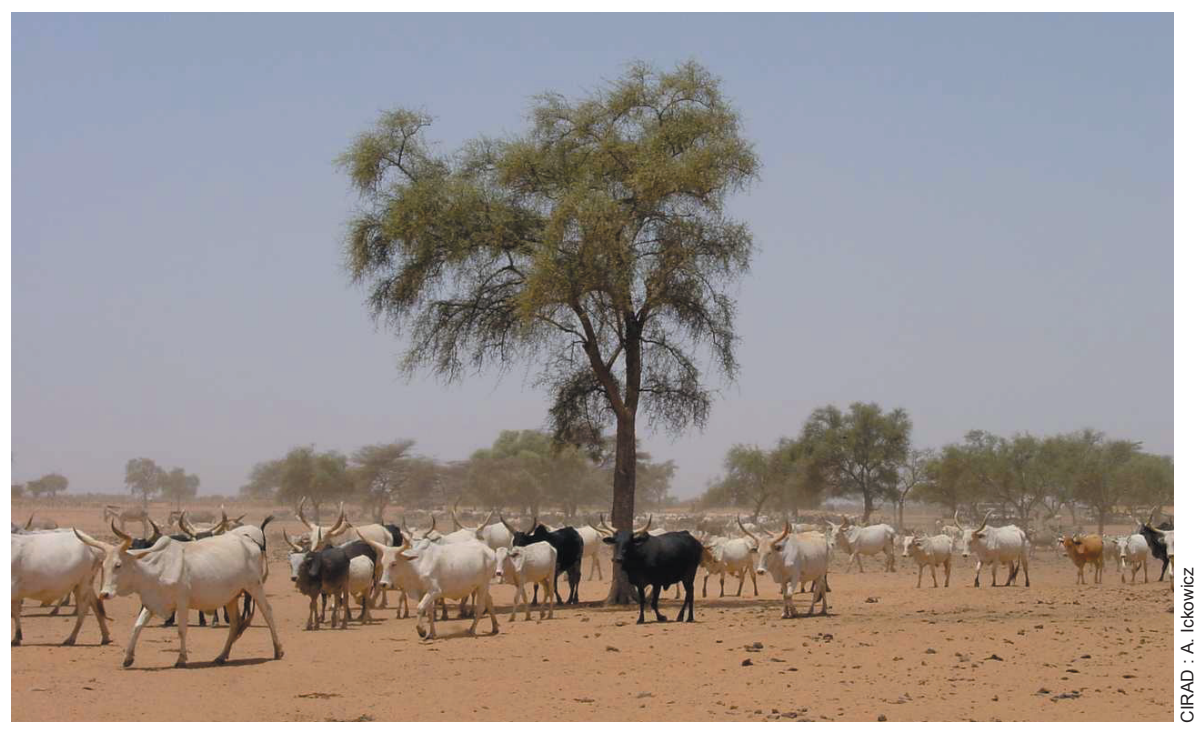

L'élevage occupe un rôle majeur sur les processus d'émission et de réduction des gaz à effet de serre responsables du réchauffement climatique.

L'augmentation rapide des productions animales au cours des dernières décennies dans les pays en développement et émergents a forcément conduit à un changement parfois radical des modes de production et par voie de conséquences des types et des niveaux d'impact sur l'environnement tels que les pollutions et la gestion des effluents.

Les ressources en eau et les ressources fossiles sont désormais des facteurs essentiels au développement des sociétés humaines et des enjeux partagés entre divers secteurs d'activité. L'élevage apparaît moins consommateur d'énergie fossile que d'autres secteurs d'activité économique, mais contribue fortement à l'appauvrissement des ressources en phosphate. Il est également un utilisateur important d'eau douce, si l'on inclut la production des aliments consommés par les animaux. Cette dernière problématique est d'ailleurs extrêmement dépendante des changements climatiques en cours. Les précipitations seront modifiées, certaines zones devenant plus arides, comme en Europe, au Maghreb ou au Brésil. En zone équatoriale et dans certaines zones tropicales humides, les précipitations pourraient augmenter mais les saisons sèches pourraient dans le même temps s'allonger et être plus sévères. L'incertitude demeure pour certaines zones comme le Sahel.

Le maintien de la biodiversité est un des autres grands enjeux majeurs auquel doit faire face l'humanité. De multiples processus de modifications environnementales sont en cause, pouvant aller jusqu'à la dégradation. Il est difficile d'isoler l'implication de l'élevage bien qu'il joue un rôle direct et indirect important à travers la gestion des habitats, leur altération et parfois leur surexploitation, l'expansion d'espèces envahissantes, les conséquences sur la biodiversité et la faune sauvage. Néanmoins, dans de nombreuses régions du monde, l'élevage d'animaux domestiques repose aussi sur des interactions positives avec le maintien de diverses formes de biodiversité à différentes échelles.

L'ensemble alimente une discussion sur les grandes tendances pour l'avenir : quels scénarios sont envisageables pour concilier l'amélioration des performances environnementales avec la mise en place de systèmes d'élevage assurant la réponse aux besoins alimentaires des populations et la durabilité économique et sociale des productions animales?

\section{1 / Elevage et environne- ment : une histoire déjà ancienne}

A l'origine, l'élevage était fondé sur l'utilisation des ressources naturelles par des animaux domestiqués, selon des pratiques d'élevages acquises et transmises empiriquement. L'animal était tributaire des ressources et la régulation entre ressources et élevage était naturelle ; telle était par exemple la situation de la plupart des systèmes pastoraux des régions chaudes jusqu'aux 
années 60 ou 70, et encore maintenant pour beaucoup d'entre eux, car dans les environnements incertains, les charges en bétail atteignent rarement des niveaux susceptibles d'endommager les ressources (Behnke et al 1993, Scoones 1995). Les progrès significatifs en santé animale, l'apport d'aliments produits par l'agriculture et le perfectionnement des techniques de production ont permis de limiter les variabilités et les aléas du milieu, d'améliorer la productivité des troupeaux et d'intensifier les systèmes de productions animales. Les élevages intensifs, surtout de volailles et de porcs se sont organisés et développés. La production de viande de poulet a été décuplée de 1961 à 2009 et celle de porc quadruplée. Ce faisant, dans de nombreuses situations, l'animal s'est trouvé de plus en plus déconnecté et indépendant des ressources de son environnement immédiat, et a bénéficié pour son alimentation de produits agricoles obtenus sur des terres arables parfois lointaines, sans prendre en compte les pollutions que cela induisait. Selon le degré de dépendance de son mode d'élevage, l'éleveur s'est trouvé plus ou moins incité à veiller à la persistance et à la qualité des ressources naturelles à sa disposition : plus le système était artificialisé, plus la recherche de l'efficacité technique et économique plaçait au second plan les questions environnementales. Si les atteintes à l'environnement de chaque élevage pouvaient paraître négligeables à l'échelle de l'exploitation, leur accumulation dans le temps et leur somme dans l'espace à l'échelle du bassin, de la région, des continents, se sont avérées considérables.

A compter des années 70, les impacts environnementaux des élevages ont alerté l'opinion publique et ont été attestés par des scientifiques. Dans les pays développés à forte densité de population humaine et animale, l'attention a été essentiellement circonscrite aux atteintes les plus visibles par leurs effets ou les plus facilement quantifiables, tant des élevages eux-mêmes que des cultures destinées à l'alimentation animale : principalement la pollution des nappes phréatiques par les nitrates et les pesticides et l'eutrophisation des rivières et des lacs associée aux épandages d'engrais et aux effluents d'élevages intensifs. Toutefois, les recherches entreprises pour réduire le recours aux engrais azotés et aux phosphates ont été d'abord motivées par le renchérissement de l'énergie lié au premier choc pétrolier de 1973. Le développement de la lutte intégrée dès les années 70 pour limiter le recours aux pesticides a pour sa part été lié aux incertitudes sur leurs effets toxiques, suite à la mise en évidence des dégâts occasionnés par le DDT
(Carson 1962) puis à son interdiction. Cette inquiétude devait conduire en 2001 à la convention de Stockholm sur la limitation des polluants organiques persistants. Dans les pays en développement, les premiers questionnements sur les interactions entre élevage et environnement ont été liés au développement numérique des ruminants en Afrique suite aux actions sanitaires, et à l'accélération considérable dans les régions équatoriales de la déforestation, notamment en Amérique latine où elle a permis l'extension de l'élevage.

Dans des zones plus arides, les graves épisodes de sécheresse au Sahel en 7273 puis en $81-82$ ont non seulement fragilisé les sociétés touchées, mais aussi fait apparaître de fortes dégradations de la végétation et des sols dans les zones subarides, accentuées par le surpâturage, et ont révélé des menaces de désertification (Boudet 1972).

La prise de conscience collective des atteintes à la nature et des risques que les sociétés faisaient courir aux ressources naturelles a conduit à la tenue, en 1992, de la Conférence Internationale des Nations Unies pour 1'Environnement et le Développement de Rio de Janeiro. Les grandes Conventions internationales qui ont suivi sur le changement climatique, la biodiversité et la désertification ont engagé les États à réorienter leurs objectifs et à adapter leurs stratégies nationales. Dans le domaine de l'élevage, secteur particulièrement visé pour ses impacts environnementaux, il en est résulté une grande frilosité des organismes de financement pour le soutien de nouveaux projets dans les pays en développement. Il était devenu difficile aux bailleurs de fonds de financer des opérations qui risquaient d'être critiquables pour leurs effets néfastes et durables sur les ressources naturelles. Ce fut la naissance d'un dilemme entre les réponses à l'accroissement de la demande alimentaire dans les pays du Sud et l'opinion publique des pays occidentaux découvrant les vertus du développement durable. Soucieux d'éviter qu'un développement sans soutien et sans régulation n'aggrave les dommages et pour des considérations économiques et sociales, un petit groupe d'experts et de bailleurs s'est constitué dès 1993 pour faire la lumière au niveau mondial sur les interactions entre l'élevage et l'environnement. Devenue en 2000 l'initiative pluri-institutionnelle et internationale LEAD (pour Livestock, Environment and Development), le groupe est désormais hébergé par la FAO puis intégré dans la Division de Production et Santé Animale. L'analyse des impacts environnementaux de l'élevage par grandes régions et par systèmes de production
(De Haan et al 1997) suivie de leur évaluation au niveau global (Steinfeld $e t$ al 1997) a mis en évidence les principaux points chauds. Ensuite des projets de recherche-développement ont testé divers modes d'intervention.

Le rapport de la FAO (Steinfeld et al 2006) a créé un choc dans le milieu des productions animales. Il montrait la responsabilité de l'élevage dans les problèmes d'environnement les plus pressants, à savoir le réchauffement de la planète, la dégradation des terres et de la biodiversité, la pollution de l'atmosphère et des eaux, et pointait la responsabilité particulièrement forte des PED et des pays émergents (déforestation équatoriale, désertification, menaces sur la biodiversité), les pays de l'OCDE montrant un certain nombre d'avances en matière de régulation sur le contrôle de la pollution des eaux, des sols et de l'air. Les principaux impacts de l'élevage étant admis, la controverse s'est alors développée sur les moyens de les limiter. Un état des lieux récent et une prospective sur l'élevage (FAO 2009) rappelle le grand essor actuel de ce secteur, avec un accroissement considérable de la demande en produits animaux, et son absolue nécessité alimentaire et socio-économique pour les populations pauvres. Il insiste sur le besoin d'appliquer des stratégies d'atténuation des impacts environnementaux et sur le rôle crucial des interventions publiques.

Le développement de l'élevage horssol dans les zones agricoles à faible densité humaine a amené à la transformation de nombreuses terres agricoles peu productives en zones d'élevage, bouleversant ainsi l'utilisation des terres en Asie du Sud-Est notamment (Vu et al 2007). En revanche, en Afrique subsaharienne, l'élevage reste actuellement peu industrialisé, et en Inde la production animale reste encore majoritairement le fait de petits élevages en zone rurale (Gerber et al 2010). L'aquaculture, pour sa part, s'est répandue en particulier dans des lacs et des zones de mangrove (FAO 2006), son développement est encore relativement récent et se caractérise par des dynamiques d'intensification rapides.

Une nouvelle analyse détaillée a été produite très récemment par la FAO, l'Université Stanford en Californie, l'Université de Berne avec la collaboration d'instituts comme le CIRAD ou l'ILRI. Ce travail important, paru sous le titre "Livestock in a changing landscape»" (vol 1: Steinfeld et al 2010, vol 2: Gerber et al 2010), confirme et développe l'argumentation sur les impacts des différents systèmes d'élevage sur l'environnement, mais donne une vision 
Tableau 1. Évolution des habitudes de consommation dans les pays en développement (en kg par personne et par an).

\begin{tabular}{|l|c|c|c|c|c|}
\hline & $\mathbf{1 9 7 0}$ & $\mathbf{1 9 8 0}$ & $\mathbf{1 9 9 0}$ & $\mathbf{2 0 0 0}$ & $\mathbf{2 0 0 3}$ \\
\hline Céréales & 145 & 159 & 170 & 161 & 156 \\
\hline Viande & 11 & 14 & 19 & 27 & 29 \\
\hline Lait & 29 & 34 & 38 & 45 & 48 \\
\hline
\end{tabular}

Source : FAO, in Steinfeld et al 2006.

régionalisée des solutions, en mettant en exergue leur diversité. L'élevage est considéré sous un angle plus large, dépassant le simple objectif alimentaire: il prend en compte le rôle économique et social important que joue l'animal pour une grande partie du genre humain, les bienfaits des produits animaux sur la santé humaine mais aussi leur responsabilité en santé publique, et bien sûr les impacts des systèmes d'élevage sur les ressources. Le tableau 1 donne un aperçu de l'évolution du recours aux productions animales dans l'alimentation humaine des pays en développement sur les 40 dernières années, bénéfique en particulier au développement des enfants. Néanmoins, l'Afrique subsaharienne et le Moyen-Orient restent en dehors de ces améliorations nutritionnelles (tableau 2), tandis que s'étendent les impacts sur leurs ressources.

L'avenir de l'élevage tel qu'il se dessine (Bruinsma 2003) comprend un recours croissant aux produits animaux dans l'alimentation humaine, le développement notable des productions intensives, surtout de viandes blanches et d'œufs. Si ces tendances présentent le risque d'une pression croissante sur les écosystèmes fragiles, elles impliquent en même temps le besoin de décisions appropriées des secteurs public et privé pour contrôler les risques environnementaux et sanitaires.

Tableau 2. Apport alimentaire en protéines d'origine animale en grammes par personne par jour (d'après Steinfeld et al 2006, Source FAOStat).

\begin{tabular}{|l|c|c|}
\hline & $\mathbf{1 9 8 0}$ & $\mathbf{2 0 0 2}$ \\
\hline $\begin{array}{l}\text { Afrique } \\
\text { subsaharienne }\end{array}$ & 10,4 & 9,3 \\
\hline Moyen-Orient & 18,2 & 18,1 \\
\hline $\begin{array}{l}\text { Amérique latine } \\
\text { et Caraïbes }\end{array}$ & 27,5 & 34,1 \\
\hline $\begin{array}{l}\text { Asie en } \\
\text { développement }\end{array}$ & 7,0 & 16,2 \\
\hline $\begin{array}{l}\text { Pays } \\
\text { industrialisés }\end{array}$ & 50,8 & 56,1 \\
\hline Monde & 20,0 & 24,3 \\
\hline
\end{tabular}

\section{2 / Elevage, dégradation et durabilité des milieux}

Les impacts environnementaux du développement de l'élevage, en particulier au Sud, sont en grande partie liés aux pratiques agricoles supportant la mise en place et le fonctionnement des systèmes d'élevages. Elles comprennent notamment le défrichement, l'irrigation, la fertilisation, l'usage de pesticides, l'utilisation d'énergies fossiles, etc. Pour autant, l'élevage apparaît aussi comme la principale activité agricole lato sensu capable de mettre en valeur une partie importante des terres, y compris non cultivables et constitue donc une source de services considérable.

La dégradation des écosystèmes peut se définir comme une réduction de ces services, une régression de leur capacité à se reproduire et en conséquence à produire durablement. La dégradation des terres agricoles est particulièrement préoccupante car la baisse de productivité qu'elle provoque entraîne en retour une perte des habitats naturels liée à l'extension des terres agricoles. Au sein des grandes régions tropicales, les dynamiques de dégradation des terres identifiées actuellement et en lien plus ou moins direct avec l'expansion de l'élevage sont la désertification des zones sèches (Afrique et MoyenOrient principalement) et la déforestation dans certaines régions tropicales humides (Amérique latine). L'aquaculture génère aussi des impacts parfois importants sur différents écosystèmes aquatiques ou côtiers ainsi que sur les ressources en eau.

\section{1 / Dégradation et désertifica- tion des parcours}

La dégradation des zones consacrées à l'élevage concerne les principaux compartiments de ces écosystèmes que sont la végétation, le sol et les ressources qui leur sont associées (eau, nutriments...), en particulier dans les régions tropicales semi-arides et subhumides. En 1991, le PNUE estimait que depuis 1945, 20\% des pâturages mondiaux étaient affectés par une dégradation significative des sols (Steinfeld et al 1997). Selon Dregne (1994), 70\% des terres des zones sèches (arides et semiarides) seraient dégradées à des degrés divers.
La désertification est une forme de dégradation qui se produit depuis les zones arides jusqu'aux régions subhumides. L'irréversibilité des dégradations conduit à la désertification par salinisation ou par induration du sol, par érosion éolienne et hydrique ou par aridification du milieu. Le surpâturage altère profondément la végétation par disparition des ligneux et évolution de la flore herbacée (simplification au profit d'espèces tolérantes au pâturage ou non consommées, baisse de la densité du couvert et de la productivité, réduction de la valeur pastorale, Toutain et al in Steinfeld et al 2010). Ces processus sont devenus préoccupants dans certaines régions : les steppes chaudes d'Afrique du Nord et du Moyen-Orient, de façon diffuse en Afrique sahélienne et l'Ouest de l'Asie.

Les systèmes pastoraux traditionnels de ces zones sèches (période de végétation inférieure à $120 \mathrm{j}$ ) ont montré qu'ils sont capables de s'autoréguler pendant de longues périodes. Cependant, depuis quelques décennies, ils sont l'un des facteurs de désertification des terres de parcours dans les zones où la pression des troupeaux dépasse les capacités des ressources, par nature limitées. Le Sahel est une vaste région typiquement confrontée à cette situation depuis près de 40 années durant lesquelles l'évolution tendancielle vers la sécheresse et les fortes variations interannuelles ont rendu le milieu plus vulnérable (Suttie et al 2005, Brooks 2006). L'élevage est victime de ces aléas et en même temps concourt à aggraver la situation en l'absence d'ajustements de conduites. Le phénomène de surpâturage survient là où le bétail se concentre sur de longues périodes (proximité des lieux d'abreuvement, autour des zones d'habitation et des périmètres agricoles). L'augmentation des effectifs de ruminants, la réduction des surfaces de parcours et de la mobilité pastorale liées à l'extension des terres agricoles et à des règles foncières défavorables sont aussi des causes de surpâturage. Le développement de points d'eau sans règles de gestion appropriées a certes conduit à désenclaver une partie des terres arides, mais a aussi favorisé leur surexploitation.

Les pâturages des régions tropicales subhumides et humides sont moins exposés aux aléas climatiques. Une partie est cependant soumise à des phénomènes de désertification, ou à des dynamiques d'embroussaillement sous l'effet de chargements élevés et continus qui entraînent la raréfaction des espèces herbacées comme les graminées au profit des ligneux et de plantes invasives. 


\section{2 / Elevage et déforestation}

Les forêts tropicales font partie des écosystèmes terrestres les plus menacés avec 200 millions d'hectares disparus depuis 1950 (Steinfeld et al 1997) entraînant notamment la raréfaction ou la disparition de nombreuses espèces (végétales et animales) et $25 \%$ des émissions mondiales de $\mathrm{CO}_{2}$ (Steinfeld et al 2006). Les forêts sèches sont menacées partout dans le monde tant par les cultures vivrières et industrielles que par l'élevage de ruminants. Mais c'est dans les zones tropicales humides que l'impact des productions animales sur la déforestation est le plus marquant, particulièrement en Amérique latine (perte de 1\% par an depuis 1970 du massif forestier initial) et de façon ponctuelle en Afrique Centrale et en Asie du Sud-Est, là où le ranching se développe aux frontières de la forêt tropicale. La mise en place et l'intensification de pâturages et de cultures sur des terres nouvellement défrichées ont été largement incitées par la croissance des productions animales dans les systèmes d'élevage intensifs de ruminants et de monogastriques.

En Amazonie brésilienne, le cheptel bovin est passé de 5 millions de têtes en 1970 à 200 millions 40 ans après, utilisant plus de $500000 \mathrm{~km}^{2}$ de prairies issues de déforestation, soit 70 à $80 \%$ de la déforestation totale (Tourrand et al 2004, Wassenaar et al 2006). Une partie plus faible mais significative des surfaces déforestées est consacrée au soja destiné à l'alimentation animale, principalement des porcs et volailles (Prudencio da Silva et al 2010). Cette conquête s'est réalisée avec peu de savoir-faire et la forêt laisse place à des systèmes herbagers extensifs répondant le plus souvent à une stratégie essentiellement foncière, en partie à l'origine d'une dégradation, d'un abandon ou d'une sous-utilisation qui sont toutefois partie intégrante du système d'élevage. Certains auteurs (Veiga et al 2004, Dias Filho 2005) estiment que plus de $50 \%$ des pâturages issus de déforestation ont un potentiel fourrager fortement détérioré (par rapport à un état initial de mise en place).

Cela ne veut pas dire pour autant que ces pâtures sont abandonnées, les repousses de ligneux peuvent par exemple être gérées avec le feu (écobuage) et les terrains cédés à des entreprises de ranching qui vont les restaurer. Seules 20\% des terres déforestées en Amazonie seraient ainsi réellement «abandonnées»), c'est-à-dire sans utilisation agricole secondaire post pâturage. Une partie est colonisée par des forêts secondaires (MMA 2001, in Tourrand et al 2004). Ce processus de déforestation lié à l'élevage bovin semble actuellement considérablement ralenti en Amazonie brésilienne du fait de la mise en place d'une politique incitative et répressive par le gouvernement. Les pressions se réalisent en aval directement auprès des grandes entreprises de distribution qui ne peuvent désormais commercialiser que la viande certifiée «non issue de déforestation». Ceci conforte les schémas de mise en valeur actuelle des zones agricoles en Amazonie forestière où les exploitants sont tenus de conserver en forêt une proportion minimum des terres qui leur sont allouées en fonction de la localisation. Il existe un zonage qui délimite des zones de «conservation» à $80 \%$ et des zones de «consolidation» à 50\%. D'autres mesures participent à cette nouvelle dynamique, telles que la création d'immenses réserves et parcs devant les fronts pionniers, l'informatisation et le contrôle des actes notariés immobiliers, etc. (Poccard-Chapuis et al 2010).

\section{3 / Autres dégradations liées à l'élevage}

La demande croissante d'aliments pour les élevages intensifs de monogastriques se traduit également par une altération de la fertilité du sol (excès de nutriments dans les sols des régions d'élevages intensifs importatrices d'aliments du fait de l'épandage des effluents, et a contrario une baisse de fertilité dans certaines régions productrices et exportatrices d'aliments). En Asie, les crises sanitaires et les épizooties récentes ont par exemple incité les autorités locales à relocaliser les élevages porcins et avicoles à la périphérie des villes (c'est aussi une volonté de rapprocher les zones de production et de consommation). Au Vietnam, l'extension des surfaces agricoles dédiées à la culture du maïs destinée à l'alimentation animale entraîne une utilisation intensive des terres alluvionnaires dans les zones de delta. En Thaïlande, la culture industrielle du manioc, notamment pour alimenter le secteur industriel de la volaille, contribue à épuiser les sols et favorise l'érosion des surfaces agricoles (Howeler et al 2001). Il faut toutefois noter que l'utilisation des terres pour les cultures destinées à l'alimentation humaine pose les mêmes problèmes que lorsqu'elles sont destinées aux productions animales.

Le développement de l'aquaculture intensive a, dans certains cas, causé des dommages aux écosystèmes côtiers et aux ressources en eaux douces. Dans l'Indo-Pacifique notamment, on observe une conversion d'une partie de ces écosystèmes en étangs d'aquaculture. Dans certaines parties de l'Asie, la culture du paddy a été remplacée sur des milliers d'hectares par l'élevage de crevettes à plus haute valeur marchande, ou bien les rizières ont vu leur productivité réduite par la salinisation causée par les entreprises d'aquaculture avoisinantes. On estime qu'aujourd'hui $87 \%$ de la mangrove ont été détruits au Bangladesh à des fins d'aquaculture de crevettes, 50 à $65 \%$ en Thaïlande, $50 \%$ aux Philippines, $80 \%$ au Vietnam, 50\%

Photo 2. Paysage agro-sylvo-pastoral de l'Est du Burkina Faso. La succession saisonnière des usages, agricole puis pastoral, permet le maintien d'une végétation arborée (arbres hors-forêt) relativement importante.

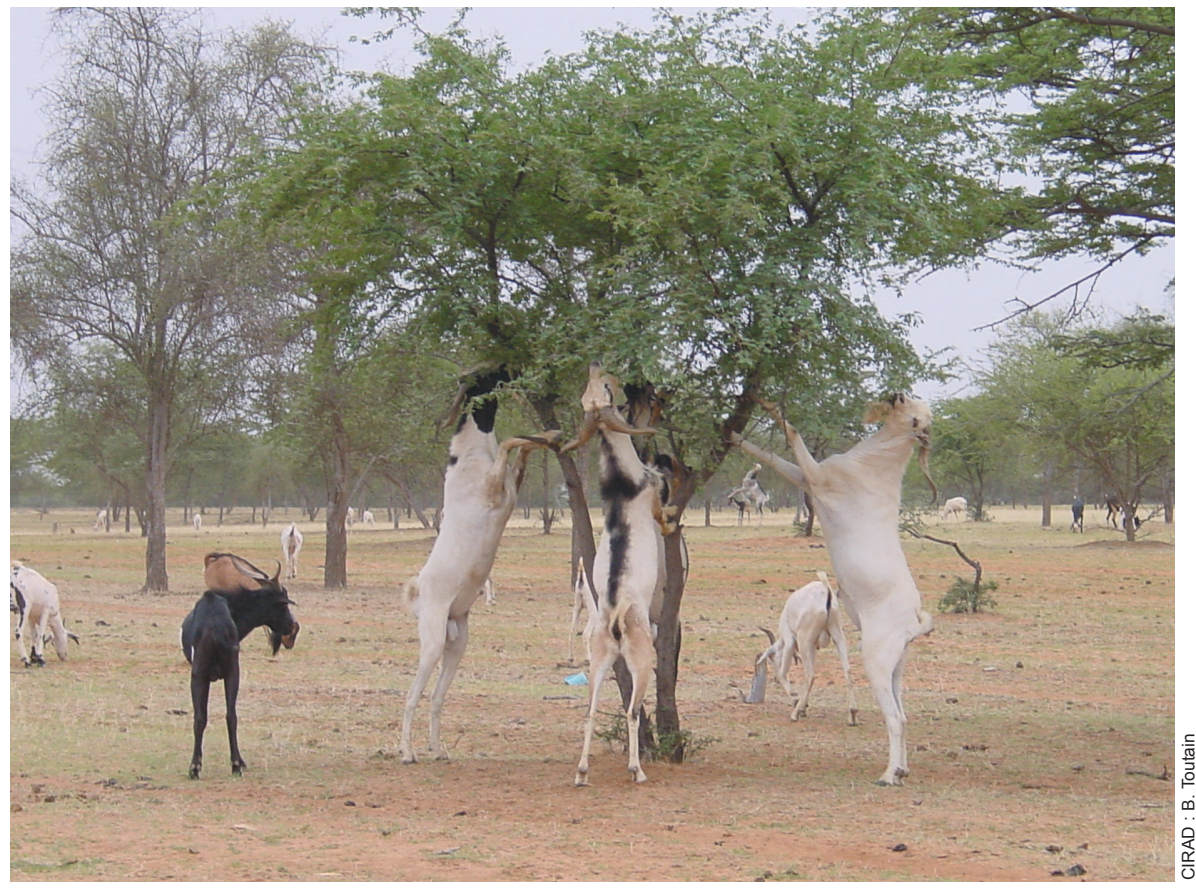


en Equateur etc. (Thornton et al 2003). Les mangroves servent de frayères et de nurseries à de nombreuses espèces marines, et revêtent une importance vitale pour le maintien de l'équilibre écologique et de la biodiversité Ces destructions de milieux naturels aquatiques conduisent toutes à divers degrés à une augmentation de l'érosion des zones côtières, à leur inondation plus fréquente et à leur exposition accrue aux accidents climatiques (par exemple le tsunami de 2004 dont les effets ont été largement tamponnés dans les zones avec mangroves intactes). Elles aboutissent aussi à la réduction de leur rôle de drains naturels, à leur salinisation et à la suppression d'habitats pour des espèces aquatiques et terrestres.

\section{4 / Contribution possible à la durabilité des écosystèmes}

Malgré les altérations et dégradations imputables aux productions animales, il est désormais admis qu'une gestion adaptée des zones d'élevage peut apporter des services environnementaux importants pouvant bénéficier à leur propre durabilité écologique, à celle des ressources qu'ils utilisent et à celle des habitats avec qui ils sont en relation directes ou indirectes. Les surfaces exploitées par les herbivores couvrent plus du quart des terres émergées libres de glaces, auxquelles s'ajoutent $1 / 3$ des terres cultivées pour la production de fourrages et d'aliments pour animaux (Steinfeld et al 2006). C'est dire l'ampleur des enjeux environnementaux qui y sont rattachés et liés directement ou indirectement à leur gestion. Au vu de ces superficies, l'élevage apparaît d'abord comme moyen incontournable de production alimentaire et de revenus ainsi qu'un véritable «valorisateur» de surfaces.

Approximativement $40 \%$ du total des terres disponibles dans les pays en développement ne peuvent être utilisées que sous forme de pâturage ou de prairies cultivées. Il n'est pas inutile de rappeler ici que $12 \%$ des habitants de la planète vivent dans des zones où la population est presque entièrement tributaire de produits provenant des ruminants (FAO 2009). Parmi ces situations, la présence de l'élevage peut contribuer à renforcer les capacités de résilience d'écosystèmes exploitées par des herbivores domestiques. Une gestion tenant compte des interactions végétation/animal peut notamment permettre de convertir l'élevage d'herbivore du statut de facteur de dégradation à celui d'élément de régulation. A cela s'ajoute le fait que l'issue de la dégradation des parcours aussi bien en zone sèche que subhumide n'est heureusement pas systématiquement irréversible. On observe fréquemment des situa- tions de réversibilités même si elles prennent plusieurs décades. Le bétail agit en prédateur, mais les dommages s'effacent peu à peu après leur passage tant que la prédation reste modérée. Depuis les dernières décennies, malgré leur sensibilité à la désertification, les parcours arides sont considérés comme des écosystèmes potentiellement dynamiques et résilients, en particulier sous gestion traditionnelle (source FAO in De Haan et al 1997). Au Sahel, selon la NASA, la végétation atteignait en 1990 les mêmes limites nord qu'avant les grandes sécheresses des années 70 et 80 .

La réversibilité des processus de dégradation et la résilience des écosystèmes impliquent aussi d'aborder leur restauration. Pour inverser le processus de dégradation des terres, remettre en état des zones surexploitées, des investissements conséquents sont souvent nécessaires, lesquels risquent de dépasser les capacités financières disponibles ou de ne pas se révéler suffisamment rentables en regard du contexte économique actuel. Au sens strict de conservation, la restauration des écosystèmes suppose le retour d'un milieu à son état antérieur, ce qui implique de déterminer l'état et le fonctionnement des écosystèmes préexistants. Il existe une acceptation plus réaliste des objectifs de restauration: stopper la dégradation d'un écosystème, puis faciliter sa relance sur une trajectoire dynamique propre à ses fonctions (proches de ses caractéristiques initiales). Pour ce type d'opération, l'écosystème dégradé doit encore présenter des facultés de résilience d'où la nécessité d'une gestion préventive de la ressource.

Quant au débat sur l'avantage environnemental entre les systèmes d'élevage extensifs et intensifs, il n'est pas encore tranché. Pour clore ce chapitre, il parait intéressant de considérer une inversion de point de vue sur l'intensification des systèmes de productions animales : la plus grande efficience de production de ces systèmes peut contribuer à la préservation des écosystèmes naturels et de leur biodiversité en réduisant le besoin de surfaces de production. Mais rien n'est simple, car l'attractivité économique de tels systèmes peut aussi générer de nouvelles arrivées de migrants et accélérer la destruction des habitats naturels (Geist et Lambin 2002, in Steinfeld et al 2010). La question de l'empreinte écologique complique aussi ce dilemme illustré par l'exemple suivant en aquaculture : au Zimbabwe (Kautsky et al 1997), l'élevage intensif de tilapia en cages flottantes sur le lac Kariba se caractérise par une forte productivité (140 kg/an de tilapia sur un $\mathrm{m}^{2}$ de cage), au prix d'une empreinte écologique conséquente : $10000 \mathrm{~m}^{2}$ pour la production de farine de poisson, $115 \mathrm{~m}^{2}$ pour la fixation de phosphore en milieu aquatique et $900 \mathrm{~m}^{2}$ d'agroécosystème pour la production des intrants végétaux. Un élevage semiintensif de tilapia en étang à partir de déchets agricoles et d'effluents d'élevage procure un rendement piscicole bien plus faible $\left(0,47 \mathrm{~kg} / \mathrm{m}^{2} / \mathrm{an}\right)$ avec une empreinte écologique très faible. En effet, il n'a recours à aucun des écosystèmes aquatique ni agricole pour l'approvisionnement en intrants et les fonctions de production d'oxygène, de dégradation de la matière organique et de l'assimilation des nutriments sont assurés in situ, au sein de l'étang.

\section{3 / Elevage et changement climatique}

La réduction des émissions de gaz à effet de serre est un enjeu majeur de la lutte contre le réchauffement climatique ; tous les secteurs, y compris ceux de l'agriculture, doivent y contribuer. Deux types d'actions sont à envisager : diminuer le niveau des émissions de GES, favoriser le transfert et stockage de carbone dans des compartiments terrestres sous une forme stabilisée.

\section{1 / L'élevage, un des responsa- bles des émissions de gaz à effet de serre}

Trois GES sont impliqués par les activités agricoles : le méthane $\left(\mathrm{CH}_{4}\right)$, le protoxyde d'azote $\left(\mathrm{N}_{2} \mathrm{O}\right)$ et le dioxyde de carbone $\left(\mathrm{CO}_{2}\right)$. Selon les méthodes de calcul du GIEC (IPCC 2007a, b) qui n'intègrent que les émissions directement imputables au secteur agricole, celles-ci seraient responsables de 14\% des émissions mondiales de gaz à effet de serre, dont $31 \%$ seraient imputables au seul sous-secteur de l'élevage. Selon l'approche en cycle de vie (ACV) de la FAO, l'ensemble des émissions impliquées par les productions animales (y compris dans le secteur des transports, de l'industrie...) est comptabilisée. Selon cette méthode, les activités d'élevage contribueraient à elles seules à $18 \%$ des émissions anthropiques de GES (Steinfeld et al 2006). Ce chiffre tient compte non seulement des émissions directes des animaux $\left(\mathrm{CH}_{4}\right.$ entérique éructé par les ruminants, $\mathrm{CH}_{4}$ et $\mathrm{N}_{2} \mathrm{O}$ des effluents des ruminants et monogastriques) mais aussi de l'ensemble des émissions indirectes de $\mathrm{CO}_{2}$ et de $\mathrm{N}_{2} \mathrm{O}$ liées à la production et à l'acheminement des intrants (fertilisants, aliments concentrés...), et à la déforestation liée aux activités d'élevage. L'augmentation de $17 \%$ des émissions de GES issues de l'agriculture entre 1990 et 2005 est essentiellement imputable aux PED et surtout aux pays 
Figure 1. Evolutions récentes et projetées des émissions de méthane et de protoxyde d'azote par l'élevage et la riziculture (IPCC 2007b).
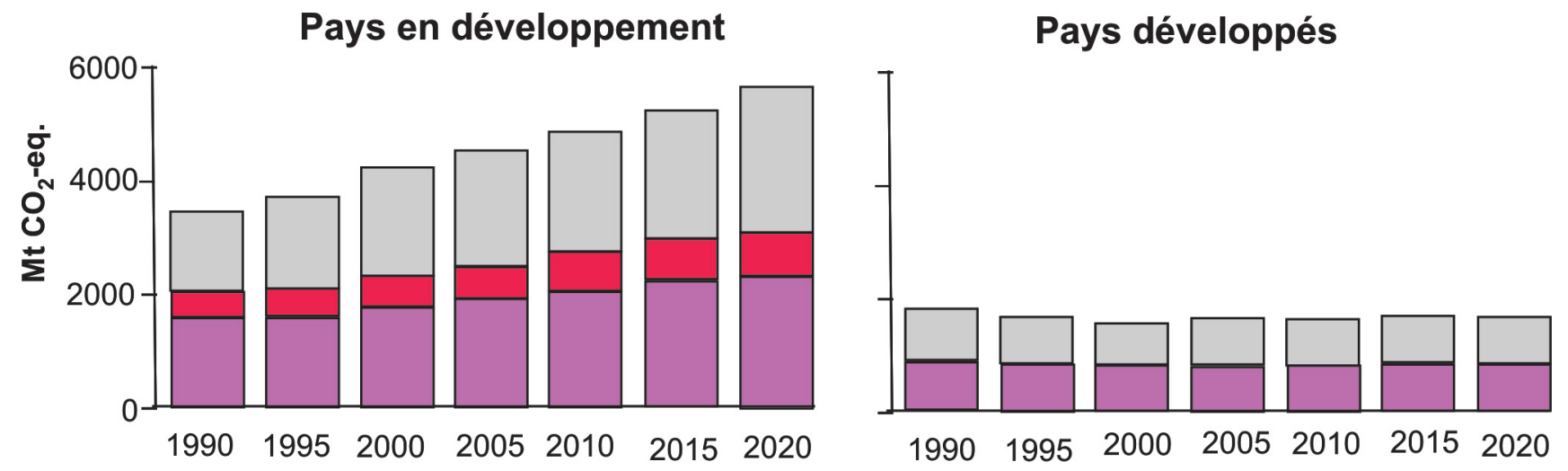

\section{$\mathrm{CH}_{4}$ animaux}

émergents (IPCC 2007b). La figure 1 montre, pour le $\mathrm{CH}_{4}$ et le $\mathrm{N}_{2} \mathrm{O}$ produits par l'élevage et la riziculture, l'évolution de la contribution des pays développés et des pays en développement.

\section{2 / Emission de GES et possi- bilités de réduction}

Actuellement ce sont les ruminants qui contribuent le plus fortement aux émissions de GES : selon la FAO (Steinfeld et al 2006), en 2004 les ruminants contribuent à $90 \%$ des émissions de $\mathrm{CH}_{4}$ d'origine animale, mais le développement de la production de porcs et volailles dans les PED et en Chine devrait accroître la part des monogastriques dans les émissions indirectes liées aux intrants.

Exprimées par $\mathrm{kg}$ de produit, lait ou viande, il est clair que les émissions de $\mathrm{CH}_{4}$ sont d'autant plus fortes que la productivité animale est faible (Martin et al 2010). Cette tendance est particulièrement nette pour les systèmes d'élevage très peu productifs liés à l'agriculture de subsistance. Ceci a conduit récemment la FAO, puis de nombreux chercheurs de pays du Nord (Etats-Unis essentiellement) et même des organismes internationaux (UNFCCC 2008) à prôner l'intensification des productions comme remède aux émissions de GES par les animaux. En fait le $\mathrm{CH}_{4}$ ne représente, en équivalents ${ }^{2} \mathrm{CO}_{2}$, à peine plus de la moitié des émissions de GES imputables à l'élevage, et l'intensification entraîne généralement un accroissement des émissions de $\mathrm{CO}_{2}$ et de $\mathrm{N}_{2} \mathrm{O}$. Dans le cas du secteur laitier, l'accroissement du potentiel de production des animaux réduit fortement les émissions par $\mathrm{kg}$ de lait jusqu'à une production de $2500 \mathrm{~kg}$ par an ; la diminution est ensuite beaucoup plus faible (de 2 à $1,5 \mathrm{~kg}$ d'équivalent $\mathrm{CO}_{2}$ par an entre 2500 et $9000 \mathrm{~kg}$ de lait par an, Gerber et al 2011). Mais si on adopte une approche liée à l'utilisation du sol et non au produit, lait ou viande, l'émission de GES par ha est plus forte en cas d'utilisation intensive des surfaces.

L'intensification des productions se traduit généralement par un accroissement de la fertilisation des prairies comme des cultures destinées à l'alimentation animale, et, dans le cas des ruminants, par un recours plus important à des aliments achetés. La volonté de maximiser la productivité entraîne ainsi des excès azotés se traduisant par des émissions accrues de $\mathrm{N}_{2} \mathrm{O}$ et un développement des transports nécessaires entre les zones de productions végétales et les élevages consommateurs, et donc des émissions de gaz carbonique. Les systèmes intensifs ont également pour conséquence la déforestation, qui se traduit, par exemple dans la zone des
Cerrados au Brésil, par l'installation du soja destiné aux ruminants, mais surtout aux porcs et aux volailles. La déforestation a des effets directs liés au brûlage, donc à la perte de biomasse, et des effets indirects liés au différentiel de puits de carbone (C) entre la forêt et les cultures ou les pâturages. Selon Steinfeld et al (2006) l'effet de la déforestation pourrait représenter le tiers des émissions de GES lié à l'élevage. Le différentiel de puits de carbone pourrait toutefois avoir été surévalué. Les études publiées jusqu'à présent font ressortir que, même si l'accroissement de productivité tend à réduire les émissions de GES par kg de produit, les différences entre systèmes d'élevage ou entre exploitations agricoles sont très importantes pour une productivité identique, les émissions allant du simple au double dans le cas des ruminants, indépendamment du niveau de production des animaux (Martin et al 2010). La quasi-totalité des publications disponibles vient des pays tempérés; il y a une besoin très fort de références obtenues dans les pays du Sud. Elles devraient confirmer que l'élevage extensif est certes fort émetteur de $\mathrm{CH}_{4}$ par $\mathrm{kg}$ de produit, mais peu émetteur de $\mathrm{N}_{2} \mathrm{O}$ et de $\mathrm{CO}_{2}$, en raison de la faiblesse des intrants.

Il est difficile de faire la part des ruminants et des monogastriques dans les

\footnotetext{
${ }^{2}$ La comparaison des émissions des différents GES passe par l'utilisation d'une unité commune l'équivalent $\mathrm{CO}_{2}$ ou l'équivalent carbone (unité d'échange des crédits d'émission prévue par le Protocole de Kyoto). Les GES sont caractérisés par leur potentiel de réchauffement Global (PRG) qui est l'unité de mesure de l'effet d'un GES sur le réchauffement climatique sur une période de 100 ans par rapport à celui du $\mathrm{CO}_{2}\left(\mathrm{PRG} \mathrm{du} \mathrm{CO}_{2}=1, \mathrm{PRG} \mathrm{du} \mathrm{N}_{2} \mathrm{O}=298, \mathrm{PRG} \mathrm{du}_{\mathrm{CH}} \mathrm{CH}_{4}\right.$ $=25$ ). Il suffit de multiplier le PRG d'un GES par la quantité émise de ce GES pour connaitre son émission en Tonne d'équivalent $\mathrm{CO}_{2}$ ( $\mathrm{T}$ C-CO $\mathrm{CO}_{2}$ ) ou Téq CO . $_{2}$ - Tonne d'équivalent CO2 d'un gaz = quantité en tonne du gaz x PRG du gaz

Les émissions de GES peuvent aussi être comptabilisées en tonnes d'équivalent carbone (TeqC) notamment pour calculer des bilans. Un kg de $\mathrm{CO}_{2}$ contient $0,2727 \mathrm{~kg}$ de carbone. L'émission d'un $\mathrm{kg}$ de $\mathrm{CO}_{2}$ vaut donc $0,2727 \mathrm{~kg}$ d'équivalent carbone

- Equivalent $\mathrm{CO}_{2}=$ Equivalent carbone $\times 0,2727\left(1 \mathrm{~T} \mathrm{C}-\mathrm{CO}_{2}=0,2727 \mathrm{TeqC}\right)$

- Pour les autres gaz équivalent carbone $=$ PRG x 0,2727.

- Equivalent carbone $=$ équivalent $\mathrm{CO}_{2} / 0,2727\left(1 \mathrm{TeqC}=3,67 \mathrm{~T} \mathrm{C}-\mathrm{CO}_{2}\right)$
} 
émissions de GES; seules des évaluations partielles sont disponibles. Le méthane entérique provient presque exclusivement des ruminants. Les émissions de $\mathrm{CH}_{4}$ et de $\mathrm{N}_{2} \mathrm{O}$ des effluents proviendraient des ruminants respectivement pour 47 et $78 \%$ (Steinfeld et al 2006). Au niveau mondial, les aliments concentrés sont consommés à $80 \%$ par des espèces monogastriques (Galloway et al in Steinfeld et al 2010). Les émissions de $\mathrm{N}_{2} \mathrm{O}$ et de $\mathrm{CO}_{2}$ liées aux cultures destinées à l'alimentation animale leur seraient donc en grande partie imputables. C'est en particulier le cas du soja.

Même si l'élevage n'est qu'un des contributeurs au changement climatique, il est indispensable de proposer des techniques de réduction des émissions («mitigation»). Celles-ci ont été particulièrement étudiées pour les ruminants. En ce qui concerne le $\mathrm{CH}_{4}$ entérique, les moyens de réduire les émissions sont divers: biotechnologies, additifs alimentaires, rations très riches en concentrés ou en certaines sources de lipides, comme la graine de lin (Martin et al 2010). Ces résultats proviennent d'essais réalisés en milieu tempéré. Ils peuvent être transposés dans les systèmes intensifs des pays du Sud. Mais il reste à explorer les solutions pour les systèmes tropicaux à base de fourrages. Il semblerait que les graminées dotées d'un métabolisme en C4 (type de photosynthèse adapté aux milieux chauds), caractéristiques des graminées d'origine tropicale à teneurs élevées en parois seraient plus productrices de $\mathrm{CH}_{4}$ que les graminées tempérées dotées d'un métabolisme en C3 (méta-analyse d'Archimède et al 2011, portant essentiellement sur des fourrages issus de prairies temporaires). En revanche, de nombreuses légumineuses tropicales sont riches en tanins, constituants ayant un effet réducteur sur la méthanogenèse. Toutefois, les tanins entraînent généralement une diminution de digestibilité des parois végétales, ce qui limite l'intérêt de leur emploi. Certaines plantes tropicales sont riches en saponines qui ont également un effet réducteur sur la méthanogenèse. Là encore, les résultats sont fragmentaires et demandent des études complémentaires. La difficulté de mise en auvre des techniques de réduction des émissions de $\mathrm{CH}_{4}$ entérique en milieu tropical fait conclure à de nombreux experts que seule la réduction du nombre d'animaux et des systèmes plus intensifs sont des moyens efficaces de réduire les émissions (Thorpe 2009). Cette vision ne tient bien sûr pas compte des services environnementaux, détaillés dans la suite du texte, et du rôle social de l'élevage.

Le $\mathrm{CH}_{4}$ des effluents est produit par fermentation anaérobie, dans les lisiers et autres déjections liquides ; il est en revanche plus faiblement produit dans les fumiers (sauf les litières accumulées) et les déjections au pâturage en raison des conditions d'aérobiose. Il existe une grande incertitude sur les facteurs d'émission du $\mathrm{CH}_{4}$ des déjections. L'IPCC (2006) propose un calcul basique dit «Tier $1 »$ affectant à l'Afrique, au Moyen-Orient et à l'Amérique latine, prises globalement, un facteur d'émission très faible, au motif que les déjections sont à forte teneur en matière sèche. Ce facteur d'émission est plus élevé pour le souscontinent indien et surtout pour les vaches laitières dans le reste de l'Asie ; mais il est beaucoup plus faible que dans les pays développés. Cette estimation extrêmement imprécise ne tient pas compte des différences entre systèmes d'élevage ; elle apparaît discutable. L'estimation plus précise dite «Tier 2» tient compte des conditions d'élevage et de traitement des déjections. Elle indique très clairement que les facteurs d'émission par animal peuvent être trois fois plus élevés dans les climats chauds qu'aux températures des climats tempérés. Ces valeurs sont souvent fournies à dire d'expert, et des travaux plus précis sont nécessaires. Toutefois elles soulignent l'importance en climat chaud, lorsque les systèmes d'élevage intensifs produisent des déjections liquides, de traiter ces lisiers au minimum par brassage pour réduire l'anaérobiose, ou de les épandre rapidement après leur production.

Les moyens de réduire, ou d'éviter d'augmenter les émissions de $\mathrm{N}_{2} \mathrm{O}$ sont, très clairement, de limiter les apports d'azote sur les cultures par les engrais au strict nécessaire, car c'est l'azote épandu en excédent qui est une source majeure d'émission, et de limiter l'apport d'azote dans l'alimentation apportée à l'animal au strict niveau de ses besoins. Il est donc important que le développement d'élevages intensifs dans les pays du Sud ne s'accompagne pas du gaspillage d'azote qui a caractérisé et caractérise encore nombre de systèmes de production au Nord; mais la connaissance encore insuffisante des besoins de fertilisation des cultures et des besoins nutritionnels des animaux en milieu tropical ne facilite pas cet ajustement. Quant aux émissions de gaz carbonique à partir de la combustion d'énergies fossiles, elles sont amenées à augmenter avec l'intensification de l'élevage. Comme pour le $\mathrm{N}_{2} \mathrm{O}$ l'ajustement optimal des intrants est à rechercher; en outre les transports terrestres pour l'acheminement des intrants sont à limiter dans la mesure du possible.

\section{3 / L'élevage et la séquestra- tion de carbone par les sols}

Sur les 2190 milliards de T de carbone $(C)$ stockés dans les écosystèmes terrestres, 1580 sont contenus dans les sols. Les forêts qui couvrent 30\% des terres émergées constituent l'écosystème qui en contiendrait le plus, soit environ la moitié (Amthor et al 1998). Les 3,5 milliards d'ha de pâturages permanents du monde se caractérisent par un stockage de $\mathrm{C}$ également très important estimé à $30 \%$ du $\mathrm{C}$ des sols du monde. Essentiellement souterrain, donc relativement stable, le potentiel de séquestration annuel au niveau de la planète (jusqu'à 0,3 milliard de T/an de $\mathrm{C}$ organique) pourrait compenser jusqu'à 4\% des émissions globales de GES (Lal 2004). Parmi cet ensemble, les pâturages tropicaux représentent un pool de stockage de $\mathrm{C}$ quasiment 2 fois plus élevé que les prairies tempérées pour lesquelles il est estimé à 182 milliards de Tonnes (figure 2).

En plus des variations climatiques, toute modification de l'usage des terres et de l'itinéraire technique des systèmes agricoles peut induire des variations de ces stocks de $\mathrm{C}$ dans les sols. Elles sont dues à divers processus : modification des apports de matière organique au sol (productivité primaire végétale, fertilisation organique), transfert de $\mathrm{C}$ sous forme solide (récoltes, érosion) ou soluble (lixiviation et ruissellement), et pertes par minéralisation $\left(\mathrm{CO}_{2}, \mathrm{CH}_{4}\right)$ de la matière organique des sols et aux pratiques des éleveurs (labour des prairies, niveau d'utilisation...). La gestion des sols apparaît ainsi comme un point clé du contrôle des stocks et flux de C.

Le stock de C immobilisé sous une prairie tempérée est estimé en moyenne à $65 \mathrm{~T}$ par hectare contre 40 sous une culture annuelle et 70 sous forêt (Arrouays et al 2002). Les sols en prairie génèrent un flux net de $\mathrm{C}$ (stockage ou déstockage) provenant de la différence entre les apports de $\mathrm{C}$ au sol (turnover des racines, des litières, déjections) et les émissions de $\mathrm{CO}_{2}$ liées à la respiration du sol. Sur un ensemble de 9 sites répartis en Europe, le bilan de carbone attribué aux prairies correspond en moyenne à un puits de 50 à $129 \mathrm{~g} \mathrm{C} / \mathrm{m}^{2}$ par an et était plus fort dans les systèmes pâturés que fauchés (Soussana et al 2007, 2010). Ces études se sont réalisées sous des gradients de type de prairies et de régime de gestion variés (chargement animal de 0,5 à 1 Unité Gros Bovin par ha et par an). Globalement, le stockage de C diminue avec l'intensité d'utilisation des prairies et est plus élevé sous gestion extensive à condition que les nutriments ne soient pas limitatifs. Lorsque l'on tient compte 
Figure 2. Stocks de carbone des principaux biomes terrestres tropicaux (tr), méditerranéens (me), tempérés (te) et boréaux (b), (IPCC 2007b)

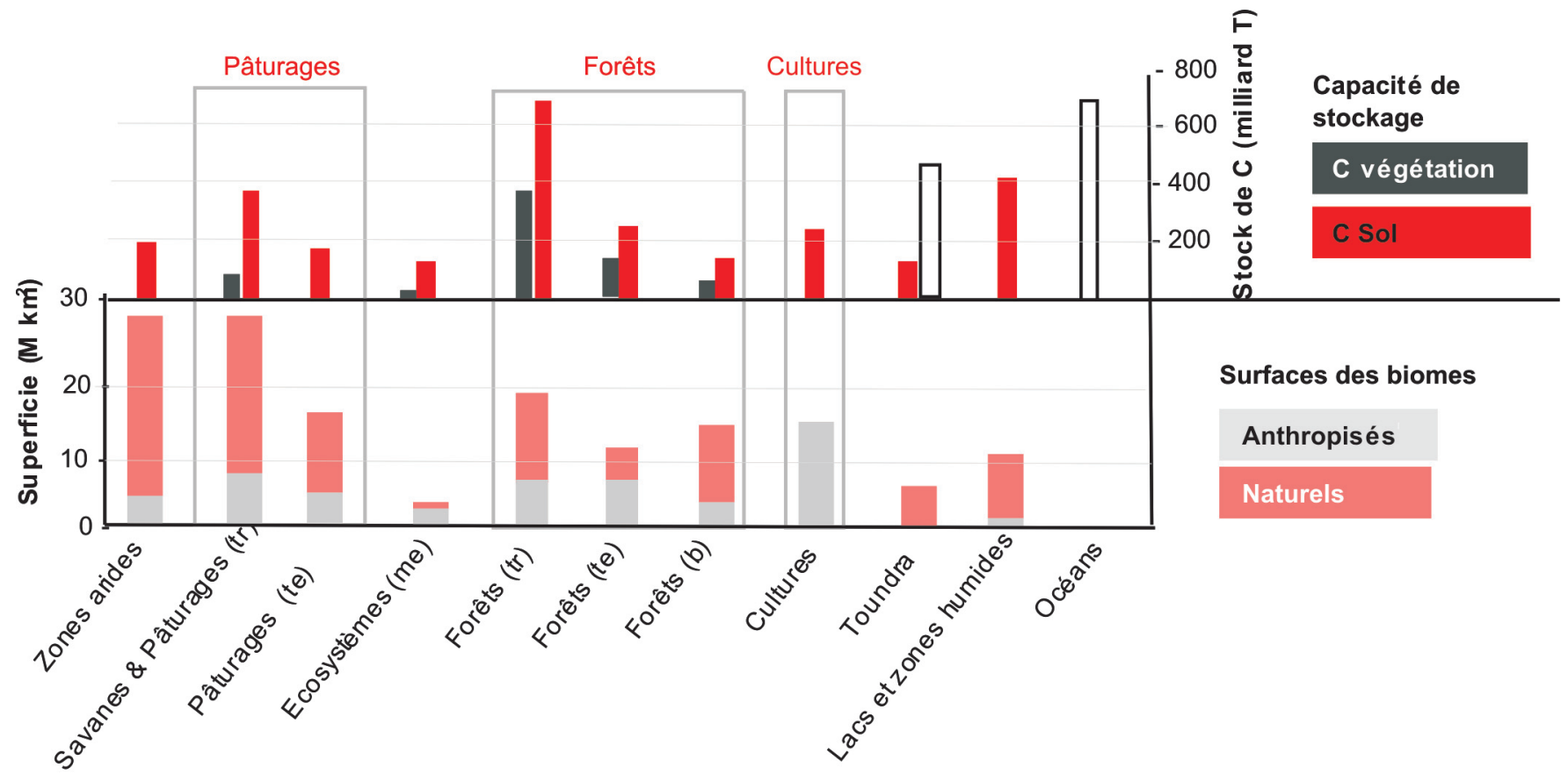

des émissions de $\mathrm{N}_{2} \mathrm{O}$ et de $\mathrm{CH}_{4}$ issues directement de la prairie et de celles provenant de la digestion à l'étable des fourrages récoltés, on détermine en équivalents ${ }^{2} \mathrm{CO}_{2}$ un bilan de GES attribué à la prairie qui représente un puits moyen de $380 \mathrm{~kg} \mathrm{C}-\mathrm{CO}_{2} /$ ha et par an (Soussana et al 2010). Toutefois, cette moyenne masque le contraste entre des prairies fauchées émettrices et des pâturages extensifs qui absorbent des GES.

En zone tropicale, ces référentiels ne sont pas encore aussi avancés aux échelles de gestion que sont la parcelle et l'exploitation dans les systèmes «fermés», et a fortiori dans les systèmes ouverts aux structures de production très différentes et aux limites géographiques moins rigides. Mais les impacts sur le cycle du C des changements de structure des écosystèmes exploités par l'élevage ont été quantifiés à des échelles larges (figure 2).

La plupart des études scientifiques admettent que le potentiel de séquestration du carbone varie selon les caractéristiques du sol et diminue avec la baisse des précipitations et l'augmentation de la température. Lal (2004) propose une gamme de séquestration variant de 0 à $150 \mathrm{~kg} \mathrm{C} / \mathrm{ha} / \mathrm{an}$ dans les régions chaudes arides, et de $100 \mathrm{~kg}$ à $1 \mathrm{~T}$ $\mathrm{C} / \mathrm{ha} / \mathrm{an}$ dans les climats humides et froids. En Afrique de l'Est, Batjes (2001 in Lipper et al 2010) estime que les parcours contiennent entre 42 et $45 \mathrm{~T} \mathrm{C} / \mathrm{ha}$ (à $1 \mathrm{~m}$ de profondeur). La dégradation et la désertification des pâturages des zones sèches peuvent réduire ce potentiel de 30 à $40 \%$. La disparition de la végétation entraîne des pertes directes de $\mathrm{C}$ aérien et souterrain par érosion et diminution (non renouvellement) des apports organiques (Asner et al 2003 in Steinfeld et al 2006). Au Sénégal, une étude de Woomer et al (2004 in Lipper et al 2010) montre que le stock varie de $12 \mathrm{~T} \mathrm{C} / \mathrm{ha}$ dans des parcours dégradés à $32 \mathrm{~T}$ C/ha dans des zones arbustives. Steinfeld et al (2006) évaluent les pertes globales de l'ensemble des zones arides touchées par la désertification entre 100 et 300 millions de T C/an. Cependant, Lal (2004) estime que les écosystèmes des terres sèches, qui représentent $40 \%$ des terres émergées, ont un potentiel «écotechnologique» de séquestration d'environ 1 milliard de $\mathrm{T}$ de carbone par an. L'auteur affirme cependant que la réalisation de ce potentiel nécessiterait «d'accomplir un effort vigoureux et coordonné de dimension mondiale en faveur du contrôle de la désertification, de la restauration des écosystèmes dégradés, de la conversion des terres à des usages appropriés et de l'adoption de pratiques recommandées de gestion des terres cultivées et des pâturages».

Dans les régions semi-arides et subhumides, le processus d'embroussaillement par des ligneux peut donner lieu à un dilemme. Considérée comme un phénomène invasif source de dégradation, cette modification du couvert végétal de certains parcours pourrait avoir comme conséquence «positive» de compenser la perte du couvert herbacé par une augmentation du $\mathrm{C}$ aérien et souterrain (Archer 1994, Van Auken 2000 cités par Steinfeld et al 2010). Mais les études sur ces processus restent contradictoires (Asner et al 2004, cité par Steinfeld et al 2010) notamment sur l'augmentation du $\mathrm{C}$ dans la matière organique du sol. De plus on pourrait craindre que les modes de gestion (souvent coûteux et difficiles) visant à limiter l'embroussaillement au profit de la production fourragère soient abandonnés du fait des incitations financières du type crédits carbone. Cela conduirait à favoriser l'altération de ces habitats par des plantes invasives.

En zones tropicales humides, les enjeux majeurs de la déforestation visà-vis de la dynamique du $\mathrm{C}$ ont conduit à des investigations importantes avec des résultats déjà significatifs. Le stock de $\mathrm{C}$ contenu dans les sols des forêts amazoniennes peut dépasser les $100 \mathrm{~T}$ (sur un total comprenant la biomasse aérienne pouvant dépasser $350 \mathrm{~T}$ dans certaines zones de Guyane d'après Blanc et al 2009). Les phénomènes de dégradation de la végétation constatés sur une partie des pâturages déforestés peuvent être liés à la perte par lessivage et surexploitation des nutriments et du C contenus dans les matières organiques du sol des pâturages implantés (Gerber et al 2010). Certaines études montrent qu'après une chute brutale suite à déforestation, ces pertes peuvent en partie être compensées après mise en place d'un pâturage bien géré. Dans certaines conditions de gestion, les stocks de $\mathrm{C}$ dans le sol peuvent se reconstituer peu à peu pour égaler voir dépasser celles des sols forestiers après 88 ans (Cerri et al 2004). Sans cautionner la déforestation et ses conséquences très négatives sur la biodiversité et sur l'effet de serre, cela 
laisse augurer qu'une gestion plus durable des surfaces déforestées transformées en pâturage peut s'envisager, pour mettre en place des systèmes herbagers capables d'assurer des productions fourragères et des services environnementaux.

\section{4 / Elevage, pollutions et gestion de la fertilité des sols}

L'essentiel des accroissements de productions animales provient non plus des systèmes d'élevage traditionnels mais des systèmes intensifs, notamment des ateliers hors-sol de porcs et de volailles, de fermes aquacoles, et, à un degré moindre, d'ateliers de viande bovine et de produits laitiers. Ces deux types de systèmes ont des impacts évidemment contrastés sur les pollutions émises (forme, quantité) et leur gestion.

\section{1 / L'élevage, source de pollu- tions}

Pour l'ensemble des espèces animales, les polluants et les impacts sur l'environnement sont divers. Les teneurs excessives d'azote et de phosphore dans les effluents d'élevage favorisent l'eutrophisation des eaux de surface (Chatellier et Vérité 2002), entraînant la prolifération d'algues et autres végétaux indésirables, et des déséquilibres importants de la faune aquatique, l'excès d'azote entraînant aussi la pollution par les nitrates des eaux profondes. L'ammoniac produit par les animaux et à un degré moindre les oxydes d'azote sont responsables de l'acidification de l'air, avec des conséquences notables sur la végétation. Certains métaux lourds utilisés en alimentation animale comme promoteurs de croissance ou pour leur activité antibactérienne (zinc et cuivre, à un degré moindre cadmium), essentiellement en élevage porcin ou avicole (Bonneau et al 2008), ou pour la transformation des produits animaux (usage du chrome en tannerie) peuvent contaminer les eaux et constituer en aval un risque pour la santé humaine et les organismes vivants. La pollution par des pesticides très variés utilisés pour produire les aliments du bétail peut être lourde de conséquences : dans les pays en développement des pesticides interdits dans les zones tempérées sont encore employés en raison d'un coût plus faible et d'une réglementation moins stricte liée à une prise de conscience insuffisante de leurs risques environnementaux. Il en est de même des pollutions par des molécules organiques consommées par les animaux (antibiotiques, hormones...), mais les informations sur les impacts de ces pratiques sont fragmentaires. Enfin, les pollutions par des organismes pathogènes (parasites, vecteurs de parasites, bactéries, virus) sont souvent nombreuses (Tilman et al 2002). Ainsi, la présence de fosses naturelles à lisier, de rejets directs dans les cours d'eau ou les mares des villages entretiennent des réservoirs septiques dangereux pour la santé des animaux et des hommes et peuvent constituer des gîtes à moustiques vecteurs de maladies. Les pollutions des eaux et des sols par les résidus médicamenteux restent encore insuffisamment connues.

Dans les régions humides, en plus des problèmes de pollution, l'érosion hydrique accroît la turbidité des eaux de surface. Partout, les transports de matières solides par les rivières et les oueds modifient les conditions d'écoulement et de drainage naturel, avec risques de crues et d'envasement des lacs et estuaires. Les eaux chargés, ou même polluées, peuvent parvenir jusqu'à la mer et causer des dommages aux côtes et aux récifs coralliens. Dans les zones d'élevage traditionnel (élevage villageois, pastoralisme), contrairement aux zones d'élevage intensif, la conjonction de la faible concentration animale avec l'usage très limité des intrants a pour conséquence une très faible pollution, à quelques exceptions près. Ainsi en Afrique ou en Inde, à proximité des points d'eau, la pollution des eaux est liée aux déjections animales qui s'ajoutent aux déchets humains. Néanmoins, la pollution limitée dans les systèmes traditionnels est à souligner, car leur faible contribution à la détérioration de la qualité des eaux et à la pollution des sols compense leur plus forte contribution aux émissions de méthane, exprimée par kg de lait ou de viande.

La production industrielle à grande échelle, en particulier celles des monogastriques, engendre d'importants impacts environnementaux avec des conséquences locales ou régionales (grands centres urbains, zone de très forte concentration de l'élevage comme la Chine de l'Ouest ou le bassin du Gange, et en aval des lagons, des deltas) et mondiales (pollution de l'air). Ces pollutions restent encore peu maîtrisées dans la plupart des pays du Sud. Le problème devient d'autant plus important que l'élevage de porcs et de volailles s'accroît très rapidement, et que des sociétés privées des pays du Nord délocalisent des élevages dans des pays du Sud (Mexique, Brésil...) suite entre autres aux contraintes règlementaires et aux coûts de production au Nord. En Chine, en 20 ans, la population de porcs a augmenté de $50 \%$ et la productivité par animal de $100 \%$ suite au développement des élevages intensifs (Bingsheng in Gerber et al 2010). Les projections pour les années à venir, en relation avec la poussée démographique, font état d'une poursuite de la croissance de toutes les productions animales dans la grande majorité des pays du Sud, l'accroissement le plus rapide étant probablement celui des productions avicoles dans les pays émergents d'Asie (Bruinsma 2003). La déconcentration des élevages nécessiterait des politiques publiques volontaristes qui n'existent pas actuellement. A un niveau global, les impacts par $\mathrm{kg}$ de produit ne sont pas clairement liés à la productivité des animaux. En l'absence d'études dans les pays du Sud, on doit se référer à des analyses de cycle de vie menées au Nord. L'accroissement de l'apport de concentrés dans les rations de bovins à viande entraîne souvent une augmentation du potentiel d'eutrophisation (Nguyen et al 2010) mais ce n'est pas systématique.

En aquaculture, la typologie des pollutions s'effectue sur d'autres bases : la distinction ne s'opère pas sur des critères de type socio-économique (industriel/artisanal) mais sur des critères dérivant des systèmes d'élevage. On distingue une pisciculture de production (extensive et semi-intensive) où le poisson trouve la part essentielle de son alimentation à partir de la productivité naturelle/stimulée de la structure d'élevage (généralement étang) et une pisciculture de transformation (intensive et super-intensive) où les besoins nutritionnels sont couverts par l'apport d'aliments complets. Les élevages extensifs et semi-intensifs n'ont pas nécessairement le plus faible impact environnemental rapporté au $\mathrm{kg}$ de poisson produit, mesuré par analyse de cycle de vie, mais leur empreinte écologique est beaucoup moins forte que celle de l'élevage intensif (cf. $\S 2.3$ ).

\section{2 / La gestion des effluents et de la fertilité}

Le rôle de l'élevage pour le maintien de fertilité des systèmes agricoles est retracé historiquement dans de nombreux ouvrages (Vigne 2004). Le couple herbivores/pâturage dans des systèmes agraires où les jachères deviennent des prairies a été à la base des premières révolutions agricoles des temps modernes (début du XVIIIe siècle en zone tempérée). Dans les PED où le tiers des ruminants est élevé dans des systèmes d'exploitation mixte (De Haan et al 1997), des dynamiques similaires y sont présentes avec des histoires et des trajectoires différentes mais cette synergie s'avère actuellement encore plus pertinente en particulier en Afrique subsaharienne (Dugué et al 2004). En zone tropicale, la matière organique des sols 
Tableau 3. Recyclage des engrais de ferme pour différentes destinations (puits) au Vietnam (Médoc et Hillion in Porphyre et Nguyen 2006).

\begin{tabular}{l|l}
\hline Type de puits & Engrais de ferme utilisables, classés par ordre d'usage \\
\hline Etangs & Effluents des élevages de : canards $>$ porcs $>$ poulets $>$ vaches et buffles \\
\hline Jardins & Effluents liquides porcins \\
\hline Cultures annuelles & $\begin{array}{l}\text { Compost humain }>\text { compost de volailles }>\text { compost de porc }>\text { compost de vaches et } \\
\text { buffles }\end{array}$ \\
\hline Cultures pérennes & Compost de porc $>$ compost de vaches et buffles \\
\hline
\end{tabular}

soumis à la culture se minéralise très vite, en particulier dans les territoires où se développent des cultures commerciales comme le coton en Afrique ou le soja au Brésil (Bertrand et Gigou 2000). La manière la plus simple et la plus économique de gérer les effluents d'élevage est ainsi de valoriser leur potentiel fertilisant sur les cultures, ce qui s'obtient par un bon équilibre entre la quantité d'animaux présents et la capacité des sols à recevoir leurs effluents.

A des degrés divers, les systèmes mixtes agriculture-élevage permettent l'utilisation des déchets produits par l'autre activité (sous-produits, matière organique). Le plus classique est l'apport de fumier qui représente pour de nombreux pays en développement, le principal apport en nutriments pour les terres $(30 \%$ des apports azotés au niveau mondial, Steinfeld et al 2006). Dans la plupart des systèmes de polyculture-élevage des pays en développement, où les sols s'appauvrissent en nutriments (pertes évaluées de 15 à $100 \mathrm{~kg} \mathrm{~N} / \mathrm{ha} / \mathrm{an}$ ), l'élevage d'herbivores contribue à la reconstitution d'une part importante du stock minéral des sols dans les zones de cultures (De Wit et al in Steinfeld et al 1997). Grâce à une gestion adaptée des déjections, l'élevage participe ainsi au maintien de la fertilité des terroirs agricoles (Schiere et al 2002). Les transferts de fertilité sont liés à la composition minérale et organique des déjections, variable selon la nature et la quantité des aliments ingérés et aux rythmes quotidiens et saisonniers des déplacements du bétail. Pour donner un ordre de grandeur, à $100 \mathrm{~kg}$ de poids vif correspondrait une production de $1 \mathrm{~kg}$ de MS fécale par jour (Landais et Lhoste 1993).

La valeur fertilisante phosphatée des effluents est difficile à prédire, car la concentration et la maturation des engrais de ferme sont en outre extrêmement variables et les capacités des cultu- res tropicales à absorber les éléments fertilisants organiques le sont tout autant (Porphyre et Nguyen 2006). La forte variabilité des modes de gestion, de stockage et de transformation des effluents entraîne une importante diversité des produits qui en résultent (fumiers, lisiers, composts, jus de biogaz) et de leur composition en nutriments (Tuan et al in Porphyre et Nguye 2006). Le tableau 3 résume les modes d'utilisation traditionnels au Vietnam selon le type d'engrais de ferme considéré (effluents liquides ou solides, compost de porc ou de volailles) et le type de surface d'utilisation (étang ou culture, légumes ou riz...).

Lorsque les quantités de matières organiques d'origine animale dépassent les besoins et les capacités d'absorption des sols, elles deviennent source de pol-

lution. C'est le cas en zones périurbaines avec les élevages intensifs. En zone rizicole d'Asie tropicale, il peut y avoir inadéquation temporelle entre la disponibilité des effluents et les besoins essentiellement saisonniers. Dans ce cas, la réduction des pollutions à la source ou des procédés de traitement s'avère nécessaire. Certaines pratiques d'alimentation animale permettent de diminuer la fraction de phosphore excrétée dans les effluents (Bonneau et al 2008), mais les connaissances actuelles en milieu tropical sur les caractéristiques physico-chimiques et nutritionnelles des matières premières sont encore insuffisantes.

Le traitement industriel des lisiers, avec séparateur de phases à la fin de l'opération, permet de concentrer

Photo 3. Au Burkina Faso, dans la zone agro-pastorale sahélo-soudanienne des zébus peul pâturent les chaumes de mil dans un terroir cultivé. Le bétail tire parti des pailles de céréale et laisse au sol des déjections fertilisantes.

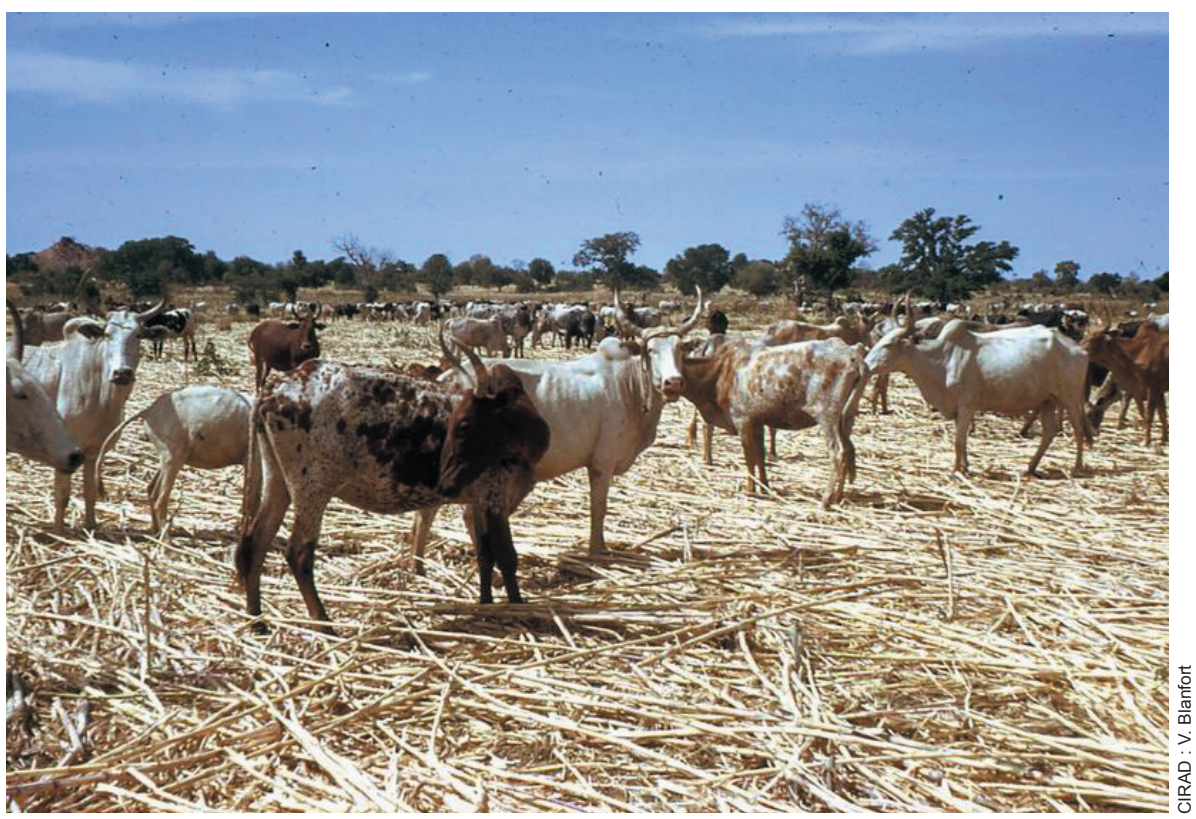


phosphore et métaux lourds en un sousproduit exportable. Mais ce traitement ayant un coût très élevé, il est rarement pratiqué dans les pays du Sud, sauf au Brésil et en Chine. Enfin, les effluents animaux peuvent également être une source d'énergie par un processus de fabrication de biogaz maintenant assez fréquent du fait de progrès techniques sur le procédé, en particulier en Asie de l'Est et du Sud (World Bank 2009).

La substitution des engrais minéraux par des engrais de ferme demeure un enjeu de durabilité (Gerber et al 2005) mais certaines contraintes pratiques (pénibilité de l'application, odeurs, connaissance de la valeur fertilisante des fumiers) et des mesures politiques dans certains pays et territoires comme les subventions aux engrais chimiques (Chine, Vietnam, Outre-Mer français) ne jouent pas toujours en sa faveur.

\section{3 / Des solutions originales pour gérer les effluents et recy- cler la matière organique}

Dans le cas des ruminants, la répartition spatiale des fèces dépend en partie du mode de conduite nocturne du bétail. En Afrique subsaharienne, il existe trois principaux systèmes traditionnels. Le parcage de nuit au piquet permet la mâ̂trise des transferts de fertilité au sol par les animaux (parcelles après la récolte, jachères). Les parcs de nuit mobiles utilisés par les Peuls consistent à parquer les animaux dans de petits enclos d'épineux sur leurs parcelles de culture ou celles d'agriculteurs. Les parcs de nuit fixes des troupeaux villageois sédentaires sont fréquemment utilisés par les agriculteurs ayant un cheptel bovin regroupé en troupeaux collectifs. Se pose alors le problème du transport des déjections accumulées vers les champs, ce qui conduit souvent à du gaspillage.

En élevage porcin, les pratiques traditionnelles que l'on retrouve dans les schémas intensifiés des pays du Sud, villageois ou industriels, consistent à effectuer une séparation de phases simplifiée en amont. Les excréments solides sont stockés et compostés pour être utilisés comme fertilisant, les eaux sales (eaux brunes) fortement diluées, peu chargées en nutriments peuvent servir à l'irrigation des cultures (EmonetDenand et al in Porphyre et Nguyen 2006).

Le recyclage direct des effluents domestiques («étangs à latrines») et d'élevages («élevages associés») tels que les porcheries et poulaillers en bordure ou en surplomb des étangs piscicoles est une tradition millénaire en Asie (tableau 3). La fertilisation de ces étangs se réalise aussi par des excré-

Photo 4. L'élevage de canards est souvent associé à la pisciculture dans le delta du Fleuve Rouge au Vietnam : les effluents des palmipèdes fertilisent l'étang et nourissent les alevins.

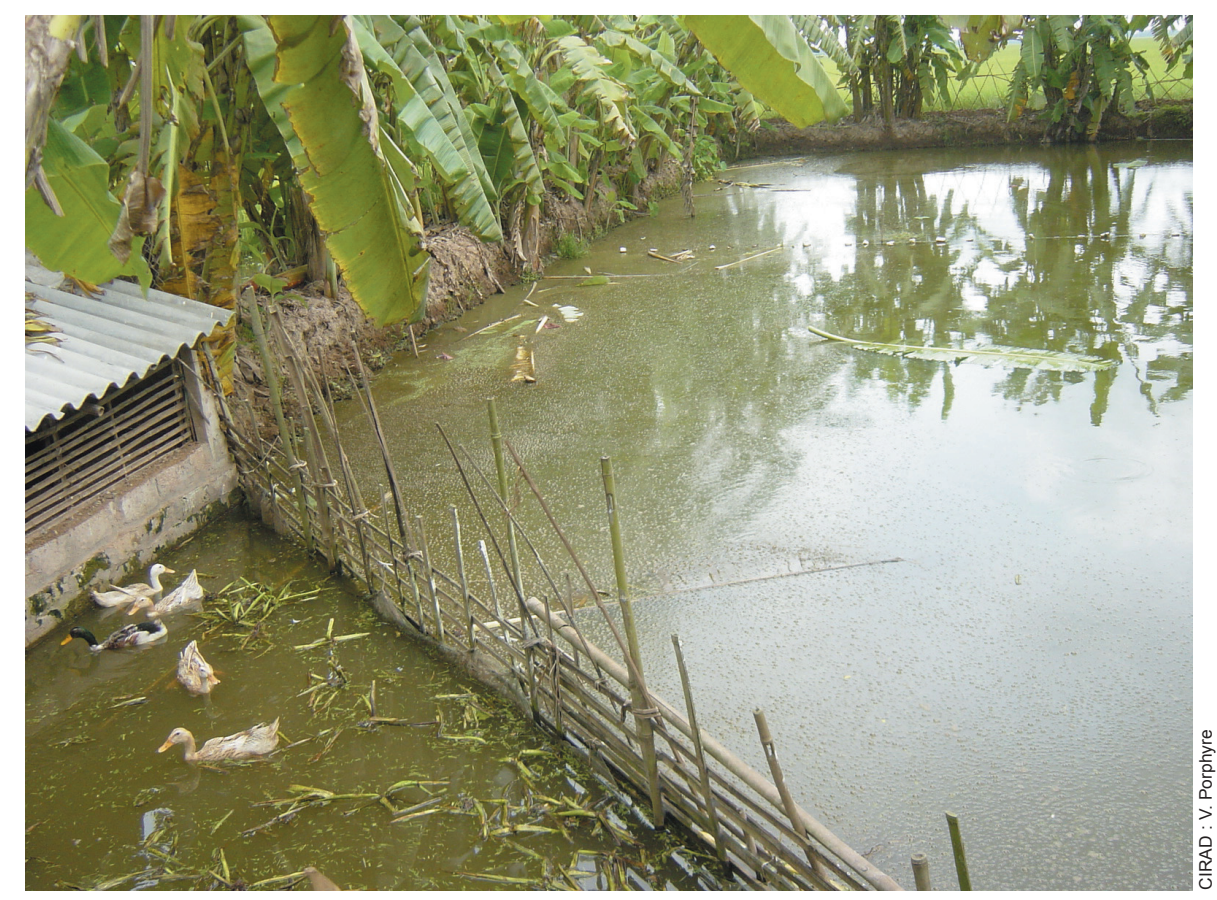

ments solides transportés depuis des élevages extérieurs. Cette pratique, encore largement répandue, a été exportée en Amérique latine et Afrique subsaharienne. Elle se caractérise par l'élevage de plusieurs espèces de poissons à régimes alimentaires différents dans le même étang, de façon à exploiter les différentes niches trophiques stimulées par la fertilisation: les rendements piscicoles varient de 5 à $15 \mathrm{~T} / \mathrm{ha} / \mathrm{an}$. L'étang peut alors être considéré comme une version aérobie des pré-estomacs des ruminants, les microorganismes se chargeant de la digestion des éléments organiques non assimilables par le poisson et constituant un apport protéique supplémentaire de haute valeur alimentaire (Schroeder 1978).

La combinaison d'élevages de monogastriques ou ruminants et de poissons réalise la dépollution d'une espèce par une autre. Un autre service environnemental attribué à l'élevage porcin dans les pays du Sud repose sur la valorisation des déchets organiques urbains et des eaux grasses. L'élevage porcin dans les pays du Sud offre un service environnemental comparable en valorisant les déchets de cuisine et de restaurants et les eaux grasses, malgré les risques sanitaires et zoonotiques associés à de telles pratiques (Madec et al 2010). On peut étendre cette notion de service environnemental de l'élevage à la valorisation des coproduits agro-industriels par les porcs et les ruminants : le lactosérum des industries fromagères, les résidus d'alcool de riz ou de tofu en Asie, les drêches de brasserie ou les écarts de triage des plantations de bananes ou de canne à sucre.

\section{5 / Elevage, ressources en eau et ressources fossiles}

Dans les régions chaudes, le partage de l'eau est désormais un enjeu de développement essentiel qui doit aussi tenir compte des besoins de fonctionnement des écosystèmes pour continuer d'assurer les divers services attendus (pour l'Homme et pour l'environnement global). L'élevage est fortement utilisateur d'eau mais il convient de nuancer les estimations globales souvent très critiques vis-à-vis du secteur par les formes d'eau utilisées (contenues dans les sols et la biosphère ou dans les réserves de surface et souterraines). Au regard des ressources fossiles, la part de consommation de l'élevage est souvent bien moindre que celle d'autres secteurs d'activité. Pour autant dans beaucoup de régions chaudes, l'eau est une ressource limitée à gérer avec parcimonie, parfois difficile à extraire et le changement climatique accentuera ces problèmes. Les besoins vont sans doute s'amplifier, notamment en Afrique subsaharienne et en Amérique latine. 
Figure 3. Cycle global et répartition de la ressource en eau douce entre l'eau "verte» (disponible pour les plantes) et l'eau "bleue» (alimentant les nappes souterraines et réserves de surface).

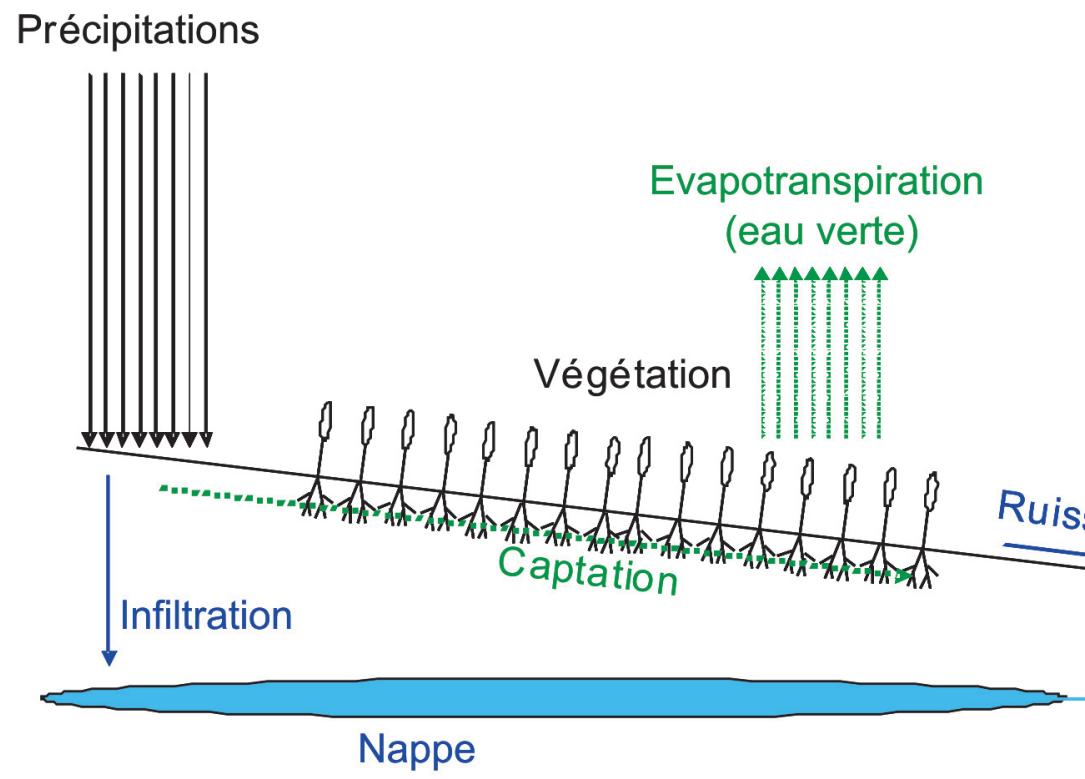

\section{1 / Elevage et ressources en eau}

L'eau douce utilisée par l'élevage est prise en compte sous deux formes (figure 3). L'eau dite verte est contenue dans les sols et dans la biosphère ; ses flux sont entretenus par l'apport des précipitations et par les sorties par évapotranspiration (qui regroupe l'évaporation par le sol nu et la transpiration par les plantes) et par ruissellement vers l'eau bleue. L'eau verte utilisée par l'élevage est mesurée par l'évapotranspiration des surfaces servant à l'alimentation animale en agriculture pluviale. L'eau dite bleue est trouvée dans les nappes souterraines et de surface (lacs, rivières) ; elle provient des précipitations, de l'infiltration et du ruissellement et s'épuise par évaporation et par consommation. Elle est utilisée pour l'abreuvement des animaux, l'irrigation des cultures destinées à l'alimentation du bétail, le fonctionnement et le nettoyage des bâtiments, et en aval de la production animale, le fonctionnement des laiteries, abattoirs et tanneries.

Le secteur de l'élevage est fortement utilisateur d'eau, mais il s'agit en très grande majorité d'eau verte. La consommation effective d'eau bleue par l'élevage (incluant les industries d'aval comme les laiteries et les abattoirs) représente environ $8 \%$ de la consommation totale par l'Homme. Les évaluations globales dont on dispose actuellement révèlent que l'élevage utilise $15 \%$ de l'évapotranspiration des cultures irriguées, un tiers de celle des cultures pluviales et $68 \%$ de celle des pâturages permanents et parcours (Steinfeld et al 2006). Au bilan, la quantité d'eau utilisée par l'élevage représenterait environ
$10 \%$ des précipitations sur l'ensemble des terres émergées (Deutsch et al in Steinfeld et al 2010). Mais les chiffres de la bibliographie sont très variables. Ainsi certains auteurs considèrent que le pâturage n'induit pas de consommation d'eau verte, puisque l'évapotranspiration potentielle (ETP) diffère peu de celle de zones naturelles non pâturées.

Les impacts directs de l'élevage sur les cycles de l'eau ne portent pas toujours à conséquence. L'utilisation d'eau verte par la végétation ne pose problème que si elle contribue à réduire la disponibilité en eau bleue, par limitation $\mathrm{du}$ ruissellement et de l'infiltration. L'irrigation (des cultures fourragères, des cultures consacrées à l'alimentation animale) perturbe le cycle de l'eau bleue, mais, mises à part les questions d'alimentation de mers intérieures ou de salinisation des sols, c'est surtout la déplétion des nappes qui peut être préoccupante. Dans les zones arides notamment, les prélèvements excèdent parfois la réalimentation naturelle (Maghreb, Péninsule arabique, Asie Centrale), et dans certaines zones humides, l'abaissement des nappes modifie l'écosystème.

Quant aux évaluations sur l'efficience de l'eau, c'est-à-dire la quantité d'eau nécessaire pour la production d'une unité de produit alimentaire, on reproche couramment à l'élevage une efficience de l'eau très inférieure à celle de la production végétale. Selon Falkenmark et Rockström (2004), il faudrait $0,5 \mathrm{~m}^{3}$ d'eau pour produire $1000 \mathrm{kcal}$ de nourriture d'origine végétale et $4 \mathrm{~m}^{3}$ pour l'équivalent d'origine animale. En 2009, le rapport de la Banque Mondiale (World Bank 2009) procède à une extrapolation à une échelle mondiale d'estimations chiffrées de l'efficience d'utilisation de l'eau pour la production de viande et de lait (nombre de litre d'eau pour $1 \mathrm{~kg}$ produit). Il est montré que l'ensemble des besoins du secteur est supérieur aux ressources mondiales. Ces chiffres doivent être considérés avec beaucoup de précautions car ils ont le défaut de prendre en considération l'eau verte de manière globale et non régionalisée, et surtout de ne pas tenir compte de l'utilisation du sol. Les problèmes d'eau doivent se raisonner à l'échelle d'un territoire pour rapporter l'ETP à l'ha, et non au kg de produit. Exprimée ainsi, l'ETP des herbages est similaire à celle des cultures. D'autre part, l'élevage extensif permet une valorisation économique de l'eau pluviale d'espaces incultes ou non cultivés. Un deuxième niveau de critique tient au fait que l'on ne tient pas compte de la valeur nutritionnelle par rapport aux besoins diététiques; les aliments d'origine animale sont une source de protéines plus que d'énergie, ce qui rend difficile la comparaison de leur consommation en eau.

L'élevage a des impacts indirects sur les flux de l'eau s'il modifie les rapports entre infiltration et ruissellement. En zones sèches, la dégradation des sols par surpâturage (cf. § 2.1) réduit l'infiltration et affecte notablement le bilan hydrique dans l'ensemble de leur épaisseur, ainsi que les flux superficiels et souterrains dans l'ensemble du bassin. $\mathrm{Au}$ Sahel, les parties hautes s'appauvrissent et se dessèchent par érosion et ruissellement, tandis que les bas de ver- 
sants s'enrichissent en eau et en éléments fins. De telles modifications dues à l'élevage résultent aussi de changements d'utilisation des sols et de couvert végétal (déforestation, défrichements).

Un des enjeux à très court terme concerne les économies d'eau à réaliser dans les différents systèmes d'élevage, notamment en fonction des contraintes climatiques. Ces économies devront porter non seulement sur les systèmes de production (moindre recours à des aliments nécessitant l'irrigation) et les systèmes d'élevage (place de l'herbe dans l'alimentation, gestion du pâturage), mais aussi sur les pratiques culturales favorisant l'infiltration de l'eau dans le sol et limitant par là même l'érosion. Une certaine attention devra donc être portée aux conséquences des changements d'utilisation des sols.

Les services que l'élevage offre en rapport avec l'eau recouvrent divers domaines et enjeux. La traction animale est utilisée en zones arides pour l'exhaure de l'eau et pour son transport. Les réseaux de forages par les programmes d'hydraulique pastorale au Sahel ont créé des lieux de vie et de fixation de petites communautés diversifiées. Dans certaines zones humides et marécageuses tempérées et tropicales, à défaut de protection totale, destiner l'usage du sol à l'élevage sur prairie porte bien moins de dommages à la biodiversité qu'une conversion à l'agriculture après un programme d'assainissement.

\section{2 / Elevage, ressources en minéraux et en énergie fossile}

La part de consommation de l'élevage est certes significative pour les phosphates et les sources d'énergie, mais limitée pour le potassium et le carbonate de calcium. Dans le cas des phosphates, les réserves mondiales, correspondant au minerai extractible à un coût économiquement raisonnable, seraient suffisantes pendant une centaine d'années, après quoi le phosphore présent dans les déchets urbains et les effluents d'élevage devraient être massivement recyclés (Castillon 2005). Au niveau mondial, la moitié du phosphore transporté par mer dans les engrais ou les aliments se rapporte à l'élevage (IFA non daté), et les productions d'engrais et d'aliments du bétail représentent respectivement 80 et $5 \%$ de l'utilisation du phosphore (12\% pour les détergents). Bien que la consommation d'engrais phosphatés ait été multipliée par 3 durant les 40 dernières années, alors qu'elle l'a été par 8 pour les engrais azotés, on peut penser que l'intensification des productions animales afin de répondre à la demande des pays émergents entraînera une poursuite de cette orientation qui était jusqu'ici le fait des pays du Nord. Il est clair que pour toutes les espèces animales, les systèmes à faibles intrants et/ou recyclant les effluents d'élevage sont beaucoup plus économes de cette ressource naturelle.

La consommation d'énergie fossile par les activités d'élevage a de multiples origines : carburant des engins agricoles et des camions pour le transport des intrants depuis le lieu d'extraction ou de production jusqu'à la ferme, électricité et chauffage des bâtiments d'élevage, des usines de production d'aliments, d'engrais, de pesticides, et processus industriels, amortissement énergétique du matériel. L'élevage industriel pour le lait, la viande et les oufs, qui s'est développé et est appelé à se développer encore dans les pays émergents (et à un degré moindre dans les pays en développement), est fort consommateur d'énergie. Les études réalisées essentiellement en milieu tempéré montrent que la consommation énergétique des élevages calculée par unité de produit est en moyenne peu variable en fonction du niveau de productivité. En revanche, pour un même niveau de productivité par animal, les systèmes les plus autonomes (moins d'achats d'aliments, moins de fertilisants) apparaissent beaucoup moins consommateur d'énergie (Basset-Mens et al 2009). Mais cela dépend bien entendu très fortement des caractéristiques de chaque système : niveau d'autonomie, nature des intrants, et éloignement de leur lieu de production. En milieu tropical, les références chiffrées font défaut, mais les systèmes extensifs traditionnels tels que le pastoralisme et les élevages villageois de volailles, de porcs et de petits ruminants, fondés sur l'exploitation de ressources locales non payantes et des coûts minima, sont de fait très faibles consommateurs d'énergie même au regard de productivités faibles. Les opérations sont en effet essentiellement manuelles et les circuits de commercialisation courts.

L'élevage industriel pour le lait et la viande est fort consommateur d'énergie. Il est à noter que la traction animale, outre ses avantages économiques, constitue une importante source d'énergie dans les systèmes d'exploitation mixte des PED où elle est utilisée sur environ la moitié des terres cultivées. Au niveau mondial, pour la culture irriguée, les animaux de trait fournissent entre 25 et $64 \%$ de l'énergie nécessaire (De Haan et al 1997). Des externalités positives sur les sols, telle l'amélioration de la structure physique, sont aussi signalées (World Bank 2009).

\section{6 / Elevage et biodiversité(s)}

Les considérations sur la diversité des espèces vivantes et sur leur variabilité génétique sont inséparables de celles sur les écosystèmes. Ces trois niveaux imbriqués de la biodiversité traversent une crise sans précédent en interagissant entre eux. Aucune région du monde n'est épargnée mais les zones tropicales sont particulièrement concernées en tant que réservoirs majeurs de cette biodiversité. Les animaux domestiques sont généralement des espèces étrangères aux écosystèmes des lieux où ils sont élevés et ils ne doivent leur survie en grand nombre qu'aux interventions de l'Homme pour les nourrir, les protéger, organiser leur reproduction et adapter leurs capacités génétiques. Ils interagissent avec les écosystèmes naturels à des degrés divers, soit directement du fait de leur présence ou de leur activité, soit indirectement (modification du milieu pour l'alimentation animale) et ce depuis des millénaires.

\section{1 / Altération des habitats écologiques}

La fragmentation et la destruction des habitats terrestres entraînent une perte de biodiversité dans les zones tropicales, en particulier au niveau des forêts, où l'élevage de ruminants est impliqué notamment en Amérique du Sud. Les zones tropicales humides abritent à elles seules $50 \%$ des espèces existantes sur seulement $7 \%$ de la surface terrestre. En Amazonie, Wilson (1989) estimait déjà la disparition de 11 à 16 espèces par jour suite à la déforestation. Or, dans cette écorégion l'élevage se révèle le facteur direct principal d'altération de la biodiversité (Malhi 2008), par la mise en place de pâturages ou par l'implantation de cultures destinées à alimenter du bétail dans d'autres régions du monde. D'autres impacts moins radicaux que la destruction d'habitat sont liés à l'intensification et la spécialisation des systèmes de production animale en zones tropicales. Ils conduisent à l'artificialisation des milieux (élimination des espèces natives et introduction d'espèces exotiques) et à la fragmentation des écosystèmes et à leur pollution qui sont autant de facteurs directs de perte de biodiversité (Steinfeld et al 2010).

Les pollutions terrestres et aquatiques causent aussi des pertes de biodiversité, certaines impliquant l'élevage. Les polluants ont des effets directs lorsque leur concentration atteint des seuils critiques, entraînant l'affaiblissement ou la mort d'espèces végétales ou animales, voire la disparition d'écosystèmes particuliers (cas des lagons). Les phénomènes d'eutrophisation peuvent aussi 
entraîner la prolifération d'organismes indirectement nuisibles pour les écosystèmes, comme les algues.

La surexploitation des ressources vivantes peut entraîner des pertes massives de biodiversité en particulier pour les poissons. Le secteur de l'élevage joue un rôle important dans la pression globale exercée par la demande en poisson. En 2006, un tiers du volume total des captures mondiales (92 MT) était destiné à l'alimentation animale et à l'industrie (FAO 2008). Sur ce tonnage, $70 \%$ des farines et $90 \%$ des huiles produites sont utilisés en aquaculture intensive ce qui aboutit parfois à une situation où certaines espèces de poissons à niveau trophique élevé consomment plus de poissons qu'elles n'en produisent : $5 \mathrm{~kg}$ de poisson sauvage pour produire $1 \mathrm{~kg}$ de poisson marin, 3 pour produire $1 \mathrm{~kg}$ de saumon ou de crevette (Naylor et al 2000).

Pour leur part, dans de nombreuses régions du monde, les herbivores d'élevage ne sont pas considérés comme intrusifs dans les écosystèmes, mais comme éléments de ces systèmes : les altérations qu'ils sont amenés à occasionner sont alors liées à des perturbations extérieures, climatiques par exemple : dans les zones sèches des régions chaudes, des pertes de biodiversité ont résulté de sécheresses exceptionnelles qui ont entraîné la régression de certaines plantes consécutivement à la désertification.

\section{2 / Les espèces végétales exo- tiques et envahissantes}

Selon le Millenium Ecosystems Assessment (2005), les espèces envahissantes constituent la seconde cause de régression de la biodiversité dans le monde. L'ampleur des phénomènes invasifs peut être considérable dans les zones de pâturage où les plantes envahissantes ont la faculté de prendre la place des espèces fourragères du fait de leur agressivité et de leur inappétibilité pour le bétail, ce qui entraîne de lourdes conséquences sur la viabilité écologique et économique des systèmes concernés qui peuvent inclure des habitats naturels (Blanfort et al 2009).

Dans les systèmes extensifs des zones sèches et des savanes subhumides, en particulier en Afrique (cf. § 2.1), le bétail peut ouvrir la voie à des plantes opportunistes qui profitent de l'hétérogénéité des couverts herbacés des savanes pâturées. Mais la situation est particulièrement préoccupante dans les systèmes pâturés tropicaux humides et subhumides. L'invasion d'arbustes et de plantes à feuilles larges menace les zones de savanes africaines (De Haan et al 1997) à travers des dynamiques d'embroussaillement qui sont parfois issues de la suppression des feux, éléments de gestion indispensables au maintien des zones de savanes qualifiées de pyroclimax par Daget et Godron (1995).

En Afrique tropicale humide, Chromolaena odorata (une Asteraceae produisant plusieurs millions de graines par pied dispersées par le vent) a probablement été introduite comme plante de couverture et dégrade fortement les couverts fourragers de plus du tiers des terres de parcours où elle limite la biodiversité des ligneux (Huguenin et Déat 1993). Dans les zones équatoriales américaines, près de la moitié des pâturages artificiels issus de déforestation est le lieu de processus invasifs qui entraînent une baisse significative de la contribution spécifique des espèces fourragères en dessous de $40 \%$ selon certaines études (Veiga et al 2004, Huguenin 2008). Cependant des stratégies d'aménagement du territoire et un pilotage adapté aux aléas climatiques conduisent à établir des systèmes herbagers amazoniens durables et notamment résistants aux pressions des espèces végétales envahissantes (Huguenin et al 2010).

Les territoires insulaires du Pacifique, vulnérables aux invasions biologiques et souvent détenteurs d'une forte spécificité biologique, constituent un autre exemple typique de perte de biodiversité. Le problème atteint des proportions considérables dans les zones exploitées par l'élevage qui comptent plus de 500 espèces d'adventices (Blanfort et Orapa 2008) dont certaines sont des pestes végétales majeures. A noter que dans les milieux insulaires, les mammifères domestiques introduits peuvent eux-mêmes devenir invasifs avec des impacts dramatiques sur la végétation endémique ; c'est le cas des chèvres aux îles Marquises, ou des cerfs en NouvelleCalédonie (Barré et al 2005).

Il faut ajouter à ces quelques exemples l'introduction volontaire d'espèces végétales accompagnant souvent le développement de l'élevage. L'usage de plantes fourragères introduites, a priori sélectionnées sur leur capacité à se maintenir et se reproduire, devient parfois une source d'invasion pour le milieu environnant. Pendant des décennies, l'Australie a organisé des programmes d'introduction d'espèces fourragères avec un bilan très négatif : $5 \%$ d'espèces effectivement utilisées, $13 \%$ devenues envahissantes, chiffre qui pourrait atteindre $81 \%$ dans l'avenir selon Lonsdale (cité par Steinfeld et al 2010).

\section{3 / Interaction de l'élevage avec la faune sauvage}

La compétition directe ou indirecte entre faune et élevage est à l'origine de situations conflictuelles entre les pasteurs et les gestionnaires des réserves de faune. C'est ainsi que les règlements de la plupart des aires protégées excluent un usage partagé des pâturages, voire même des points d'eau, avec le bétail. (Toutain et al 2004). Les éleveurs protègent leur bétail en éloignant ou tuant les animaux prédateurs comme les grands fauves dont les prédations au Kenya peuvent atteindre $3 \%$ de la valeur économique du troupeau (Steinfeld et al 2010). Les éleveurs éloignent aussi les herbivores sauvages s'ils représentent des concurrents pour les ressources. La lutte contre des parasites ou des animaux vecteurs de maladies peut atteindre d'autres espèces non cibles : par exemple les bousiers ou les abeilles sont menacés par les usages de pesticides pour le bétail. Les impacts sur la faune sont parfois involontaires et peu perceptibles: ce sont par exemple les perturbations dans les zones de nidification d'oiseaux, la dégradation des végétations boisées le long des cours d'eau qui sont les refuges de nombreuses espèces, les impacts sur la macrofaune du sol, ou l'appauvrissement des mangroves pâturées qui compromettent la reproduction de nombreuses espèces marines.

Cependant les interactions entre la faune sauvage et le bétail sont très complexes et de nombreuses études scientifiques valident la complémentarité faune sauvage-bétail domestique sur la ressource pâturée et sur la diversité des espèces elle-même (De Haan et al 1997). On reconnaît les avantages que procure l'élevage aux écosystèmes par rapport à d'autres utilisations du sol. Certains systèmes extensifs utilisent souvent des espaces «naturels», sans intervention directe notable si ce n'est la conduite du bétail. Leur impact sur les écosystèmes est bien moindre que des défrichements pour des utilisations agricoles. De plus, les herbivores domestiques ont des comportements comparables aux herbivores sauvages par rapport au milieu. Ils contribuent à la transformation et au recyclage de la biomasse par la fragmentation des végétaux, la digestion microbienne puis les déjections. Le maintien de paysages ouverts favorables au bétail et de végétations herbacées spontanées contribue à entretenir une riche biodiversité végétale et animale dans certains environnements.

\section{4 / Diversité génétique des espèces domestiques}

La biodiversité recouvre aussi les ressources zoogénétiques : les espèces, races et lignées d'intérêt écono- 
mique, scientifique et culturel pour l'humanité aux fins de l'agriculture, dans le présent et pour le futur. Une des questions majeures qui se pose en matière de biodiversité des espèces animales exploitées est la suivante : considère-t-on que le nombre d'espèces et de races dont l'élevage est maîtrisé et dont la production atteint un volume significatif convient pour un développement futur de l'activité dans un contexte de développement durable? Dans le monde entier, la diversité des animaux domestiques est menacée par le caractère spécialisé de la production animale moderne (en 2000, 1300 races domestiques sont éteintes où menacées selon Steinfeld et al 2006). Sur la quarantaine d'espèces domestiquées de mammifères et d'oiseaux, 14 d'entre elles fournissent $90 \%$ de la production animale mondiale (UNEP 1995 cité par Steinfeld et al 2010). Sur les 32000 espèces de poissons du monde, 230 font l'objet d'élevage, et 25 seulement contribuent à $91 \%$ de la production aquacole totale (FAOStat 2010). La globalisation et l'extension des marchés généralisent peu à peu cette tendance des pays développés vers les pays du Sud. La chute des populations porcines Mong Cai au Vietnam ou des races créoles en Caraïbes et à la Réunion est la conséquence de l'introduction massive de races à forte croissance de type Large White ou Landrace (Porphyre et Nguyen 2006). En aviculture, seules 4 firmes internationales fournissent la majeure partie du matériel génétique et exercent une pression immense sur les populations de volailles traditionnelles. Cela peut aussi entraîner une pollution génétique des populations domestiques locales, qui sont par ailleurs déjà très hétérogènes dans le contexte des pays du Sud, avec peu de races vraiment fixées.

L'introduction d'espèces exotiques à des fins d'aquaculture est très ancienne et s'est multipliée ces dernières décennies. Le nombre de telles opérations était évalué en 2006 à plus de 5600 (FAO 2008). Beverton (1992), dans sa revue globale des introductions d'espèces aquatiques, concluait que la majorité des espèces introduites s'étaient montré soit non viables, soit sans impact écologique. Une petite proportion d'entre elles s'est révélée bénéfique et quelques-unes, de nature invasive ou prédatrice, ont eu des effets destructeurs sur la faune autochtone. Il est ainsi couramment admis que la dégradation généralisée des habitats aquatiques serait susceptible de conférer aux deux espèces de tilapia $(O$. niloticus et $O$. mossambicus) le statut d'espèce invasive (De Silva et al 2006). Pourtant une étude de l'introduction des tilapias dans la région Asie-Pacifique n'a pas révélé d'impacts négatifs majeurs directs ou indirects sur la biodiversité. En Asie, aucune introduction inter- ou intracontinentale à des fins d'aquaculture n'a causé d'impacts liés à la prédation comparables aux conséquences de l'introduction de la perche du Nil dans le lac Victoria.

Pour des raisons similaires, le phénomène touche aussi la diversité génétique des cultures fourragères et des céréales destinées à l'alimentation animale (espèces dominantes maïs, soja, sorgho) suite à la Révolution Verte et se poursuit par la diffusion de variétés génétiquement modifiées.

Les environnements des PED constituent cependant un facteur de conservation des ressources zoogénétiques en privilégiant l'utilisation de races et souches locales adaptées qui présentent des résistances aux maladies ou des aptitudes de rusticité valorisables dans les conditions difficiles des petits élevages du Sud. Parmi les races africaines traditionnelles de bovins, le taurin N'Dama a développé une trypanotolérance (la trypanosomiase menacerait 50 millions de bovins dans 37 pays d'Afrique selon la FAO). Pour autant, les effectifs de cette race sont en constante régression. Le programme de sélection sur le tilapia du Nil (Genetically Improved Farmed Tilapia) développé aux Philippines durant les années 80 et 90 (Gupta et Acosta 2004) a rencontré un succès limité auprès des exploitations artisanales (qui représentent l'essentiel de la production en Asie) attachées à l'utilisation de souches traditionnelles, mais son succès s'est principalement imposé auprès des producteurs industriels (Lazard 2009).

\section{7 / Discussion}

Au-delà de l'image généralement négative véhiculée par l'élevage dans les débats en cours sur les enjeux agroenvironnementaux globaux, le bilan qui précède a tenté de prendre en compte la complexité des relations de l'élevage avec l'environnement dans le contexte des régions chaudes. L'objectif était d'apporter des nuances sur l'intensité des impacts de l'élevage, leur pérennité, leur portée régionale et leur compensation par des services. Plutôt que la recherche d'une exhaustivité irréalisable dans le cadre de cet article de synthèse, nous avons opté pour un «balayage» aussi large que possible des gammes de situations offertes par l'extrême diversité des contextes écologiques et des systèmes de production des pays du Sud.

\section{1 / Impacts des systèmes d'éle- vage pour les différentes produc- tions animales}

Le tableau 4, inspiré d'une approche présentée dans le rapport «Livestock's long Shadow» de Steinfeld et al (2006), synthétise l'ensemble des impacts et services environnementaux majeurs actuels qui caractérisent l'élevage des régions chaudes au sein des 5 grands enjeux traités dans cet article. Il se dégage une spécificité marquée entre groupes d'espèces (ruminants, monogastriques, poissons) et au sein des systèmes de production. Ceux-ci ont été limités en nombre par souci de simplification et de clarté, mais il existe une très grande diversité de systèmes dans l'ensemble des zones tropicales et arides. Globalement, le passage de systèmes traditionnels extensifs ou mixtes vers des systèmes intensifiés a eu un effet positif sur l'amélioration de l'efficacité de l'utilisation des terres et de l'eau mais des effets négatifs sur les divers processus de pollution, de consommation d'énergie et sur la diversité génétique (FAO 2009).

Par rapport aux bovins, largement évoqués dans cet article, qui représentent la principale biomasse animale des herbivores, la production des petits ruminants est en général de nature extensive et se réalise souvent dans des lieux et des conditions inadaptés à toute autre forme de valorisation. Il n'en demeure pas moins qu'ils peuvent aussi être à l'origine de dégradations des pâturages et des zones boisées notamment. Concernant les monogastriques, le secteur avicole industriel semble globalement causer moins de dommages à l'environnement que les porcins (notamment du fait d'une efficacité de transformation supérieure nécessitant moins de surfaces cultivées). Les effluents sont sensiblement plus faciles à gérer pour les volailles, car plus secs. Les litières peuvent être transportées plus efficacement et leur forte concentration entraîne une valeur marchande suffisante pour garantir une utilisation totale. Enfin, la consommation d'eau reste peu importante en ce qui concerne l'activité d'élevage. Cependant, à l'échelle des exploitations, la taille des élevages et les pratiques de gestion des effluents conditionnent les impacts des deux secteurs.

L'aquaculture se singularise par des dynamiques d'intensification assez récentes et rapides. Ainsi le rendement moyen des étangs de pisciculture chinois a été multiplié par 6 entre 1980 et 2000 ( $\mathrm{Li}$ 2003). Elle génère des impacts conséquents en amont 
Tableau 4. Principaux services environnementaux (point rouge) et impacts (carré noir) des systèmes de production animale en régions chaudes.

$\mathrm{n}$ : neutre, $\bullet / \mathbf{m}$ : faible, $\bullet \bullet / \mathbf{m}$ : modéré mais significatif, $\bullet \bullet \bullet / \mathbf{m m}:$ fort. Le rattachement de chaque impact au sein des 5 enjeux est rappelé par le numéro de $\S$ de l'article correspondant en $1^{\circ}$ colonne : 2 . Elevage et changement climatique, 3 . Elevage, pollutions et gestion de la fertilité des sols, 4. Elevage, ressources en eau et ressources fossiles, 5. Elevage et biodiversité(s), 6. Elevage, dégradation et durabilité des milieux. Les impacts et services sont déclinés pour chaque grand type de production (ruminants, monogastriques, poissons). Effets indirects (ind). Les impacts pour lesquels l'information manque ou est très imprécise n'ont pas été mentionnés.

\begin{tabular}{|c|c|c|c|c|}
\hline & & \multicolumn{3}{|c|}{ Ruminants } \\
\hline$\S$ & Impacts et services & $\begin{array}{l}\text { Pastoralisme } \\
\text { Agro-pastoralisme }\end{array}$ & $\begin{array}{l}\text { Petit élevage } \\
\text { villageois }\end{array}$ & $\begin{array}{c}\text { Ranching-système } \\
\text { herbager (en voie } \\
\text { d'intensification) }\end{array}$ \\
\hline 2 & Emissions de GES par kg de produit & $m a$ & $\square \square$ & $\mathbf{\square}$ \\
\hline 2 & Séquestration de C & $\bullet$ & $\bullet$ & $\bullet \bullet$ \\
\hline 3 & Consommation d'intrants & $\mathbf{n}$ & - & $m$ \\
\hline 3 & Pollutions eaux et sols & - & - & edra \\
\hline 3 & Fertilisation organique & $\bullet$ & $\bullet$ & $\bullet$ \\
\hline 4 & $\begin{array}{l}\text { Consommation ressources non } \\
\text { renouvelables et énergie }\end{array}$ & $\mathbf{n}$ & $\mathrm{n}$ & $m$ \\
\hline 5 & Biodiversité végétale et animale & $\bullet$ & $\mathbf{m}$ & $\square \square$ \\
\hline 5 & $\begin{array}{l}\text { Diversité génétique animaux } \\
\text { domestiques }\end{array}$ & $\bullet \bullet$ & $\bullet \bullet$ & $\mathbf{m}$ \\
\hline 6 & Dégradation sols, végétation & - & $\bullet / n$ & ex/na \\
\hline \multirow[t]{2}{*}{6} & Dégradation / durabilité des habitats & ed/ana & ed/na & edana \\
\hline & & \multicolumn{3}{|c|}{ Porcs et volailles } \\
\hline$\S$ & Impacts et services & $\begin{array}{l}\text { Petit élevage } \\
\text { villageois }\end{array}$ & $\begin{array}{l}\text { Elevage } \\
\text { villageois } \\
\text { intensifié }\end{array}$ & $\begin{array}{l}\text { Elevage périurbain } \\
\text { semi-intensif }\end{array}$ \\
\hline 2 & Emissions de GES par kg de produit & $\mathbf{n}$ & - & 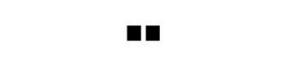 \\
\hline 3 & Consommation d'intrants & $\mathbf{n}$ & - & $m$ \\
\hline 3 & Pollutions des eaux et des sols & In & In & olna \\
\hline 3 & Fertilisation organique & $\bullet$ & $\bullet$ & e./na \\
\hline 4 & $\begin{array}{l}\text { Consommation ressources naturelles et } \\
\text { énergie }\end{array}$ & $\mathbf{n}$ & $\mathbf{n}$ & $\mathbf{m}$ \\
\hline 5 & Biodiversité végétale et animale & -In & $\square$ & - \\
\hline 5 & $\begin{array}{l}\text { Diversité génétique des animaux } \\
\text { domestique }\end{array}$ & $\bullet \bullet$ & $\bullet$ & $\mathbf{m}$ \\
\hline 6 & Dégradation / durabilité des habitats & घ & घ & घ \\
\hline
\end{tabular}




\begin{tabular}{|c|c|c|c|c|}
\hline & & \multicolumn{3}{|c|}{ Poissons } \\
\hline$\S$ & Impacts et services & $\begin{array}{l}\text { Extensif en étang } \\
\text { faible densité }\end{array}$ & $\begin{array}{l}\text { Semi-intensif } \\
\text { en étang } \\
\text { Densité } \\
\text { moyenne }\end{array}$ & $\begin{array}{l}\text { Intensif à productivité } \\
\text { modérée }\end{array}$ \\
\hline 2 & Emissions GES par kg de produit & & & an \\
\hline 3 & Consommation d'intrants & $\bullet$ & 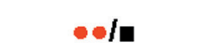 & m \\
\hline 3 & Pollution du milieu environnant & $\bullet$ & $\bullet / n$ & 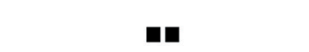 \\
\hline 3 & $\begin{array}{l}\text { Consommation ressources naturelles et } \\
\text { énergie }\end{array}$ & $\bullet$ & - & $\bullet / n$ \\
\hline 5 & Etat sanitaire des populations sauvages & $\mathrm{n}$ & $\mathbf{n}$ & $\square$ \\
\hline 5 & biodiversité végétale et animale & & - & 口I \\
\hline 5 & $\begin{array}{l}\text { Diversité génétique des animaux } \\
\text { domestique }\end{array}$ & $\bullet$ & $\bullet$ & 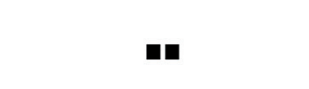 \\
\hline $5-6$ & Dégradation / durabilité des habitats & חII & I & I \\
\hline
\end{tabular}

(utilisation croissante de farines et huiles de poissons et de protéines végétales à haute valeur biologique) et en aval (impact environnemental). Cette intensification s'accompagne néanmoins d'une amélioration de l'efficience des systèmes d'élevage (cf. ci-dessous et figure 4).

\section{2 / Des solutions complémen- taires pour gérer durablement l'élevage, mais des difficultés de mise en ouvre}

Les pressions de l'élevage sur l'environnement sont en augmentation depuis quelques décennies. En contrepartie, la mise en place de services écologiques, même si elle est encore émergente, suit aussi une tendance croissante par laquelle la prise de conscience de leur existence tend à changer la vision négative de la société sur l'élevage. Au sein de ces tendances, on observe une dichotomie croissante entre un secteur moderne d'élevage intensif dans certains pays du Sud concernant surtout les productions laitières, l'élevage de monogastriques, l'aquaculture, et un secteur traditionnel relégué sur les terres les plus marginales par les cultures et les biocarburants. Plusieurs scénarios d'évolution sont envisageables, mais dans tous les cas la mise en place de modes de fonctionnement nouveaux apparaît indispensable. Il s'agit d'apporter dans les contextes des pays du Sud des solutions complémentaires assurant aux systèmes d'exploitation une viabilité économique indispensable, leur recevabilité écologique et leurs fonctions sociales. Ainsi l'instauration d'une taxe carbone au Nord (déjà en œuvre dans certains pays comme la Suède) réinvestie dans l'aide à la restauration des écosystèmes au Sud (visant à réduire la déforestation, à limiter la perte de $\mathrm{C}$ des sols, les émissions excessives de méthane, préserver la biodiversité...), pourrait contribuer à l'émergence au Sud de formes d'élevage «durables», mais modernisées à certains égards, à faible impact environnemental et capables d'assurer des services écologiques.

Le marché carbone actuel repose sur le Mécanisme de Développement Propre (MDP) qui constitue un des trois mécanismes du Protocole de Kyoto permettant aux pays développés de réduire leur émission nette de $\mathrm{C}$ (donc d'augmenter leur crédit d'émission C) en promouvant dans les PED des projets d'énergie renouvelable, d'efficacité énergétique ou de séquestration du C. Ce mécanisme repose sur des réductions certifiées des émissions (RDE) qui sont encore relativement restreintes. Pour le secteur de l'élevage, celles-ci ne concernent encore que les émissions issues de la production (récupération de $\mathrm{CH}_{4}$, traitement des déchets d'animaux de stabulation). Pour les projets de changement d'utilisation des terres, seul le boisement est éligible et peut atténuer les émissions du secteur élevage en reconvertissant par exemple des pâturages dégradés en forêts. Les autres formes d'amélioration des pâturages ne sont pas concernées (Steinfeld et al 2006). En raison de leur complexité, les activités de fixation du C dans les sols ne sont pas encore incluses dans les MDP.

Une autre option possible consiste à appliquer les coûts réels des intrants et externalités environnementales (l'eau est notamment largement sous payée). Mais le marché des coûts de l'environnement est encore balbutiant, pas toujours efficace et les initiatives politiques souvent insuffisantes ou inadaptées. De plus, le contexte de pauvreté de nombreux pays du Sud, les inégalités d'accès aux ressources naturelles, des contextes climatiques parfois très contraignants compliquent considérablement l'application de telles mesures et soulèvent évidemment des considérations morales.

Des réductions de subventions à l'élevage, options pas vraiment nouvelles, se sont parfois traduites par des réductions de dégradations à l'environnement. Cela concerne surtout les pays développés mais parfois aussi les PED ou émergents. Les soutiens aux prix affectent l'échelle de production en favorisant la spécialisation, l'utilisation d'intrants, l'expansion des surfaces (sur des terres parfois sensibles) qui engendrent des pressions sur l'environnement. Une éco-conditionnalité ou une réorientation de telles subventions vers le paiement de services environnementaux constitue une incitation changeant le rapport de l'agriculture avec l'environnement, à l'instar de ce qui est pratiqué en France et en Europe (Politique Agricole Commune). 
Figure 4. Représentation schématique de l'analyse du cycle de vie de 2 systèmes de production aquacoles : intensif en cages flottantes en Indonésie et semi-intensif en étangs côtiers aux Philippines. L'impact environnemental est proportionnel à la surface du polygone : données du projet EVAD en cours de publication (Aubin et Van der Werf 2009).
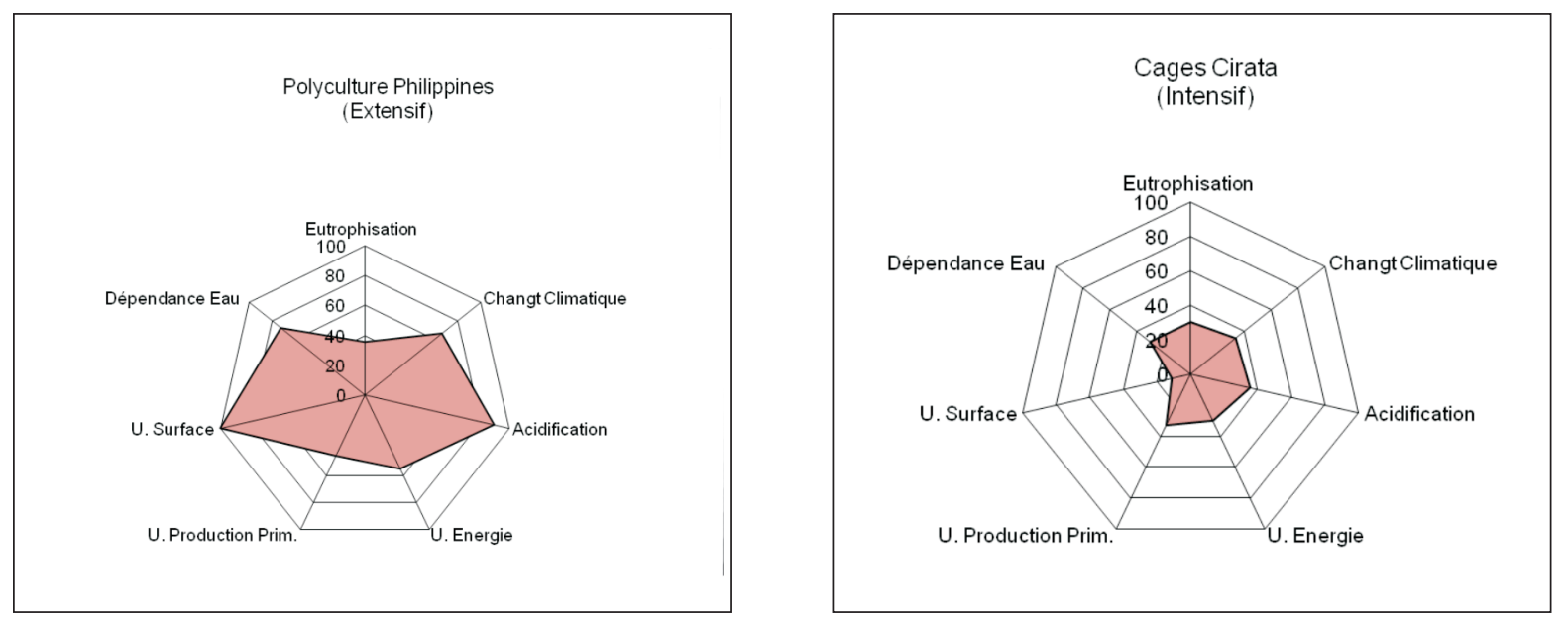

La grande question des changements climatiques est un autre facteur déterminant des évolutions des rapports de l'élevage avec l'environnement. Ceci forme d'ailleurs une boucle de «rétroaction» puisque l'élevage lui-même est en partie responsable du réchauffement climatique par augmentation de l'effet de serre. L'adaptation des systèmes d'élevage au changement climatique est un enjeu majeur à moyen terme pour la durabilité des filières correspondantes. Il s'agit d'identifier des voies d'adaptation possibles à différents niveaux (ressource alimentaire, animal, troupeau, système d'élevage) en actionnant des leviers différents (génétique, conduite d'élevage, gestion de la santé, technologie alimentaire...).

Pour les situations évoquées tout au long de cet article, des solutions existent donc en région chaude pour réduire les impacts négatifs de l'élevage et renforcer ou favoriser l'émergence de services environnementaux. Mais les obstacles à leur mise en place ne sont pas négligeables et leur levée relève souvent du long terme. Le premier obstacle, d'ordre général, est qu'il existe, parmi les producteurs, les consommateurs et les législateurs, une incompréhension de la nature et de la portée de l'impact de l'élevage sur l'environnement. Beaucoup d'impacts touchent l'environnement de manière indirecte restant ainsi moins perceptibles et souvent minimisés. Dans la majorité des pays du Sud, on constate l'absence d'un cadre réglementaire propice à l'adoption de pratiques plus écologiques, qui demeure un préalable indispensable. Franchie déjà non sans mal par les pays de l'OCDE, cette étape est loin d'être une priorité dans les pays du Sud où la lutte contre la faim, la malnutrition et la pauvreté relèguent généralement les préoccupa- tions environnementales au second plan. La levée de ce type d'obstacles est donc conditionnée en partie par le développement économique de ces zones. Pourtant le cas des pays émergents montre que les progressions en matière de développement économique ne vont pas forcément de pair avec une plus grande exigence environnementale des productions agricoles. On peut enfin citer la pression de lobbies de production intervenant parfois depuis l'extérieur dans le cas de productions géographiquement délocalisées.

\section{3 / Objectifs de développement et perspectives de recherche}

Quels schémas seront capables de concilier l'amélioration des performances environnementales avec la mise en place de systèmes d'élevage assurant la réponse aux besoins alimentaires des populations et la durabilité économique et sociale indispensable aux sociétés en développement ? Les systèmes traditionnels n'arrivent pas (plus) à satisfaire la demande croissante en produits d'élevage dans de nombreux pays en développement pour des raisons d'ailleurs parfois non liées à leurs propres capacités. L'intensification des productions apparaît incontournable à la satisfaction des enjeux alimentaires à l'horizon 2050. Cependant les voies classiques d'intensification, par simplification technique et dans un objectif de maximisation rapide ne sont plus des modèles acceptables pour l'avenir dans un concept de développement durable. Elles ont en effet abouti à dissocier complètement l'idée d'une gestion conjointe de l'élevage et de l'agriculture en générant de nouveaux impacts environnementaux. A l'opposé, et en relation étroite avec la problématique de réduction des GES et du stockage du carbone, dans les milieux à fortes contraintes du sud, la mise en synergie des deux systèmes et le développement concomitant d'améliorations utiles pour l'un et l'autre serait une voie intéressante à revisiter

Ces évolutions nécessaires posent également des questions de recherche renouvelées. Au-delà de l'effet indéniable de la déforestation et du changement du mode d'occupation des sols sur les pertes de carbone, une des voies de recherche possible consiste à définir des pratiques capables de compenser en partie les pertes en carbone occasionnées par la mise en place des pâturages au détriment de la forêt. Dans le même temps, il s'agit de maintenir un niveau de fertilité suffisant pour produire durablement du fourrage de qualité et en quantité. Des investigations scientifiques préalables doivent permettre de mieux comprendre le fonctionnement agro-écologique des systèmes déforestés tropicaux, en particulier les mécanismes de stockage du C dans le sol car ils apparaissent très liés à ceux contrôlant la fertilité des sols sous pâturage (Blanfort et al 2010, Fontaine et al 2007 in Soussana et al 2010). D'une manière plus globale, le changement climatique nécessite en premier lieu l'évaluation des impacts sur l'élevage. Ensuite, deux voies de recherche doivent être abordées simultanément: la réduction («mitigation») de ces impacts, par des voies génétiques, nutritionnelles ou technologiques, et l'adaptation des animaux et de leurs aliments au contexte de réchauffement. Bien entendu, il y a des interactions entre ces deux approches: par exemple, le réchauffement et les périodes de sécheresse peuvent entraîner un développement de dicotylédones comme les légumineuses, et ces dernières contribuent à limiter les émissions de GES. 
Compte tenu du grand nombre de facteurs à prendre en compte (abiotiques ou biotiques), une vision intégrative de la recherche apparait nécessaire au-delà de la seule approche expérimentale. Les connaissances scientifiques à l'échelle de l'écosystème (notamment sur les mécanismes pilotant son fonctionnement et sur les processus de régulation interne) doivent être couplées à une démarche de modélisation comme celle entreprise dans les études des impacts du changement climatique sur la production agricole (Gitay et al 2001).

Les services écologiques occupent une place croissante dans les questionnements scientifiques. Il devient indispensable de développer des méthodes pour analyser, quantifier, valider, certifier les services produits par différents types d'écosystèmes, et en particulier comprendre les contraintes et les opportunités pour la fourniture simultanée de plusieurs services. Ces investigations contribueront à la mise en place du paiement de ces services écologiques qui peuvent être considérés comme un moyen de combattre la pauvreté tout en répondant à de nombreux autres objectifs socioéconomiques et environnementaux essentiels. Ces questions font maintenant partie des affichages prioritaires, du niveau régional ou national au niveau des grands projets de recherche internationaux.

La mise en œuvre de solutions techniques alternatives pour la fourniture d'aliments concentrés dans les filières porcs, volailles et aquacoles constituent une autre question tout aussi impérieuse et incontournable. L'accroissement des productions animales attendu d'ici 2050 (doublement) reposera en effet en grande partie sur les monogastriques. Le maintien du système actuel de recours massif au maïs et au tourteau de soja est intenable. Il constituerait par exemple une entrave à la politique volontariste des états amazoniens pour diminuer la déforestation du fait de la pression très forte des acteurs économiques. Dans le même ordre d'idée, l'essor de l'aquaculture mondiale a engendré une forte demande de farines et d'huiles de poisson fabriquées à partir des captures de pêches dont la production stagne déjà. Une des voies de développement proposée par la recherche (Kaushik 2009) consiste à diversifier les ingrédients des aliments piscicoles, pour réduire la dépendance de l'aquaculture aux matières premières marines.

Ces différentes voies de recherche sont de type sectoriel. La recherche d'alternatives techniques indispensables à l'évolution des systèmes d'élevage nécessite une vision intégrée des différentes composantes de la durabilité de ces systèmes, et pour ce qui concerne le volet environnemental de la durabilité, des différents impacts et services environnementaux. Les ACV constituent un outil de choix qui en fournit une analyse multicritère. A titre d'exemple, le cas de l'aquaculture, récemment étudié, est particulièrement riche d'enseignements. La diversité des systèmes de production aquacole donne lieu à une gamme d'impacts environnementaux cumulant des effets directs et indirects : l'eutrophisation, l'acidification, le changement climatique, l'utilisation d'énergie, l'utilisation de production primaire nette, la dépendance à l'eau et le travail (Aubin et Van der Werf 2009). Leur approche permet d'identifier les points clés qui conditionnent les performances environnementales et suggère des voies d'amélioration. Un travail de ce type (Rey-Valette 2008) appliqué à 6 systèmes de production aquacole contrastés en Europe, Afrique et Asie a permis de faire ressortir que les facteurs alimentaires et énergétiques sont ceux qui régissent les impacts des systèmes les plus intensifs. Dans les systèmes les moins intensifs, le niveau des impacts (calculé relativement à une unité pondérale de poisson) est très lié à la productivité du système et à sa capacité à valoriser les intrants.

Cette étude a en outre, contribué à remettre en cause une idée largement répandue : la supériorité des systèmes aquacoles de type extensif en termes de durabilité (figure 4). Les choix de développement futurs de certaines filières ne sont pas aussi simples et évidents qu'il ne paraît, et cela ne peut être mis en évidence que par des méthodes d'analyse prenant en compte la complexité des enjeux agro-environnementaux.

Enfin on peut se poser la question de savoir si les demandes faites à la recherche concernant les interactions de l'élevage avec l'environnement sont différentes au Sud et au Nord?

En aval, les aspects socio-économiques apparaissent souvent très spécifiques. Concernant les processus biologiques, les thématiques et les objets sont plus comparables, donc les approches et les outils transposables avec des adaptations indispensables. L'élargissement des gammes de situations qu'offrent les régions chaudes présente aussi un intérêt notamment vis-à-vis des changements climatiques à venir. La mise en commun des recherches du Nord et du Sud offrent donc des bénéfices mutuels, ce qui n'a guère été pratiqué jusqu'à présent. Les moyens de recherche étant essentiellement au Nord, une réelle coopération Nord-Sud entre organismes de recherche est à encourager sur la base des nombreux réseaux d'alliance existant déjà.

\section{Conclusion}

L'accroissement de la consommation de produits d'origine animale et par voie de conséquence des productions animales prévue d'ici 2050 concernera pour l'essentiel les pays émergents et les PED. Cette priorité indiscutable de sécurité alimentaire et de lutte contre la pauvreté ne doit pas pour autant exclure ces pays des grands accords internationaux qui marquent désormais la marche vers une conscience globale et collective des problèmes actuels et futurs de la planète (Blanfort et al 2009). Les aides au développement des productions animales qui en découlent deviennent en grande partie soumises à l'acceptabilité des modes de production par la communauté internationale. La recherche d'un équilibre entre les fonctions de production et la préservation des autres services permettant le bien-être humain (régulation du climat et de la qualité de l'environnement, contrôle biologique, valeurs culturelles...) est un des piliers du développement durable au Sud comme au Nord.

Concernant les productions animales des régions chaudes, elles devront dans le même temps continuer à remplir un rôle vital envers les besoins alimentaires des populations et réduire leurs impacts environnementaux. Ceci constitue une spécificité des productions animales des pays du Sud, alors que pour les élevages du Nord, les enjeux sont sociétaux et environnementaux. L'autre spécificité (et difficulté) des pays du Sud consiste à intégrer dans ces problématiques environnementales les «petits paysans» (l'agriculture familiale) qui sont la grande majorité des éleveurs des régions en développement. Un vaste champ d'investigation multidisciplinaire impliquant la recherche et ses utilisateurs s'ouvre donc pour accompagner l'évolution des systèmes d'élevage des pays du Sud et leur interaction avec l'environnement. Leur complexité nécessitera notamment de s'appuyer sur le rapprochement des sciences biologiques et socio-économiques.

La capacité de la recherche à apporter des réponses peut par exemple aider à résoudre un des dilemmes du développement au Sud. Préserver l'espace pastoral, en améliorant la productivité de ses systèmes de production animale (jugée trop faible aujourd'hui en regard de ses impacts environnementaux) contribuerait au maintien de populations menacées au plan économique et social. Inversement, l'intensification des productions (s'appuyant notamment sur les monogastriques) peut nécessiter l'extension des cultures 
consacrées à l'élevage pour nourrir «les villes».

Une autre révolution agricole est donc à inventer, au sein de laquelle le développement des productions animales a tout à fait sa place. Le concept d'agriculture «écologiquement intensive» a été proposé par Griffon (2010); l'alliance de ces deux termes longtemps considérés comme inconciliables propose de trouver les moyens de produire plus et mieux en consommant moins. Elle se base sur des innovations technologiques s'appuyant sur l'écologie scientifique pour trouver les moyens d'intensifier les processus naturels. Sa performance ne se mesure pas seulement sur les quantités de biens agricoles produits mais elle tient aussi compte des services écologiques rendus («chercher un rendement par unité de biosphère»). Les termes de l'équation consistent à augmenter raisonnablement la production en limitant l'extension des surfaces et en augmentant les rendements par des méthodes non destructives de la qualité de l'environnement. C'est un concept qui reste à instruire pour les productions animales.

\section{Remerciements}

Vincent Blanfort est cofinancé par l'Union Européenne (Fonds européen de développement régional). Merci à Denis Bastianelli (Cirad) pour ses commentaires sur la filière volaille.

\section{Références}

Amthor J.S, 21 others, 1998. Terrestrial ecosystem responses to global change: a research strategy. Oak Ridge National Laboratory, Oak Ridge, TN, ORNL/TM-1998/27.

Archimède H., Eugène M., Marie Magdeleine C., Boval M., Martin C., Morgavi D.P., Lecomte P., Doreau M., 2011. Comparison of methane production between temperate and tropical forages: A quantitative review. Anim. Feed Sci. Technol., paru en ligne, doi :10.1016/j.anifeedsci.2011.04.003.

Aubin J., van der Werf H.M.G., 2009. Pisciculture et environnement : apports de l'analyse du cycle de vie. Cah. Agric., 18, 220-226.

Arrouays D., Balesdent J., Germon J.C., Jayet P.A., Soussana J.F., Stengel P., 2002. Stocker du carbone dans les sols agricoles de France. Expertise scientifique collective, INRA, 334p.

Barré N., Le Bourgeois T., De GarineWichatitsky M., Tassin J., Blanfort V., Strasberg D., 2005. Stratégies et méthodes innovantes développées par la recherche dans les territoires français du pacifique et de l'océan indien occidental pour limiter l'impact des espèces envahissantes. Proc. Caribbean Food Crops Society, 41, 89-95.

Basset-Mens C., Ledgard S., Boyes M., 2009. Eco-efficiency of intensification scenarios for milk production in New Zealand. Ecol. Econ., 68, 1615-1625.

Bertrand R., Gigou J., 2000. La fertilité des sols tropicaux. Ed. Maisonneuve et Larose, Paris, France, 397p.

Behnke R.H., Scoones I., Kerven C., 1993. Range ecology at disequilibrium. New models of natural variability and pastoral adaptation in African savannas. Overseas Development Institute, London, UK, 248p.

Beverton R.J.H., 1992. Fish resources: threats and protection. Neth. J. Zool., 42, 139-175.

Blanfort V., Orapa W., 2008. Ecology, impacts and management of invasive plant species in pastoral areas. Proc. Regional Workshop on Invasive Plant Species in Pastoral Areas. Blanfort V., Orapa W. (Eds). 24-28 november 2003, IAC/SPC, Suva, Koné, New Caledonia, 201p.

Blanfort V., Doreau M., Soussana J.F., 2009. Elevage et environnement, quels enjeux en zone tropicale ? Séminaire Enjeux et priorités de la Recherche pour l'élevage dans les pays des Sud, Paris, 2 déc. 2009. Renc. Rech. Rum., 33-41.
Blanfort V., Fontaine V., Picon-Cochard C., Klumpp K., Huguenin J., Soussana J.F., 2010. Evaluation of carbon sequestration in tropical grassland, ecological processes and farm practices in Amazonian cattle systems. Adv. Anim. Biosci., doi:10.1017/ S2040470010001111

Blanc L., Echard M., Herault B., Bonal D., Marcon, E., Chave J., Baraloto C., 2009. Dynamics of aboveground carbon stocks in a selectively loggedtropical forest. Ecol. Applic., 19, 1397-1404.

Bonneau M., Beline F., Dourmad J.Y., Hassouna M., Jondreville C., Loyon L., Morvan T., Paillat J.M., Ramonet Y., Robin P., 2008. Connaissance du devenir des éléments à risques dans les différentes filières de gestion des effluents porcins. In : Numéro spécial, Porcherie Verte. Bonneau M. (Ed). INRA Prod. Anim. 21, 325-344.

Boudet G., 1972. Désertification de l'Afrique sèche. Adansonia, 12, 505-524.

Brooks N., 2006. Changement climatique, sécheresse et pastoralisme au sahel. Question sur l'adaptation. IMPD, 12p. http://data. iucn.org/wisp/fr/documents french/climate ch anges fr.pdf

Bruinsma J., 2003. World agriculture: towards 2015/2030. An FAO perspective. Earthscan Publications, Londres, UK, 440p.

Carson R., 1962. Silent Spring. Boston, Houghton Mifflin. Edition française : Printemps Silencieux, 1963, Plon, Paris, France, 283 p.

Castillon P., 2005. Le phosphore : sources, flux et rôles pour la production végétale et l'eutrophisation. In : Numéro spécial, Le phosphore dans l'alimentation animale. Meschy F., Sauvant D., Pinot P. (Eds). INRA Prod. Anim., 18, 149-151.

Cerri C.E.P., Paustian K., Bernoux M., Victoria R. L., Mellilo J.M., Cerri C.C., 2004. Modelling changes in soil organic matter in Amazon forest to pasture conversion, using the Century model. Global Change Biol., 10, 815832.

Chatellier V., Vérité R., 2002. Élevage bovin et environnement. INRA, Dossier de l'Environnement, 23, 93-127.

Daget P., Godron M., 1995. Pastoralisme : Troupeaux, espaces et sociétés. Hatier, AUPELF-UREF, Paris, France, 510p.

De Haan C., Steinfeld H., Blackburn H., 1997. Livestock and the Environment: Finding a Balance, 186p. http://gis.lrs.uoguelph.ca/ agrienvarchives/bioenergy/download/livestock env fao.pdf

De Silva S.S., Nguyen T.T.T., Abery N.W., Amarasinghe U.S., 2006. An evaluation of the role and impacts of alien finfish in Asian inland aquaculture. Aquac. Res, 37, 1-17.

Dias Filho M.B., 2005. Degradaçao, de pastagens: processos, causas e estratégias de recuperaçao. 2ème Ed., Embrapa, Amazonia, 173 p.

Dregne H.N., 1994. Degradation and restauration in arid lands. Dregne H.N. (Ed). Lubbock, USA, Texas Technical University, USA, 289p.

Dugué P., Vall E., Lecomte P., Klein H.D., Rollin D., 2004. Evolution des relations entre l'agriculture et l'élevage dans les savanes d'Afrique de l'Ouest et du Centre. Un nouveau cadre d'analyse pour améliorer les modes d'intervention et favoriser les processus d'innovation. Oléagineux Corps Gras Lipides, 11, 268276.

Falkenmark M., Rockström J., 2004. Balancing water for humans and nature: the new approach in ecohydrology. London, Earthscan, UK, 247p.

FAO, 2006. State of world aquaculture 2006. FAO Fisheries Technical paper 500, Rome, Italie, $134 \mathrm{p}$.

FAO, 2008. Database on Introductions of Aquatic Species (DIAS). http://www.oceansatlas.com/world fisheries and aquaculture/html /resources/aqua/introspec/dias.htm\#topofdocu$\underline{\text { ment }}$

FAO, 2009. The state of food and agriculture. Livestock in the balance. FAO, Rome, Italie, $166 \mathrm{p}$.

FAOStat, 2010 http://faostat.fao.org/default. $\underline{\text { aspx?lang=fr }}$

Gerber P., Chilonda P., Franceschini G., Menzi H., 2005. Geographical determinants and environmental implications of livestock production intensification in Asia. Bioresource Technol., 96, 263-276.

Gerber P., Mooney H., Dijkman J. Tarawali S., Haan C. de, 2010. Livestock in a changing landscape. Vol. 2. Experiences and regional perspectives. Island Press, Washington, USA, 208p.

Gerber P., Vellinga T., Opio C., Steinfeld H., 2011. Productivity gains and greenhouse gas emissions intensity in dairy systems. Livest. Sci., paru en ligne, doi:10.1016/j. livsci.2011.03.012 
Gitay H., Brown S., Easterling W., Jallow B., 2001. Ecosystems and their goods and services. In: Climate change 2001: impacts, adaptation, and vulnerability. Contribution of Working group to the third assessment report of the intergovernmental panel on climate change. McCarthy J.J., Canziani O.F., Leary N.A., Dokken D.J., White K.S. (Eds). Cambridge University Press, USA, 235-342.

Griffon M., 2010. Pour des agricultures écologiquement intensives. Editions de l'Aube, 143 p.

Gupta M.V., Acosta B.O., 2004. From drawing borrad to dining table: The success story of the GIFT Project. NAGA WorldFish Center Quarterly, 27, 4-14.

Howeler R.H., Oates C.G., Allem A.C., 2001. An Assessment of the impact of cassava production and processing on the environment and biodiversity. Proc. Validation forum on the global cassava development strategy, 26-28 april 2000. FAO, Rome, Italie, 17p.

Huguenin J., 2008. Gestion des prairies amazoniennes contre les adventices en Guyane française suivant les conditions biophysiques, les pratiques agricoles, et l'organisation du système pâturé. Thèse, École Doctorale ABIES/AgroParisTech, CIRAD, INRA, Paris, France, $428 p+$ annexes.

Huguenin J., Déat M., 1993. Répartition dynamique et incidences de Chromolaena odorata (L) R.M. King et Robinson dans huit pays d'Afrique de l'Ouest: Bénin, Cameroun, Centrafrique, Côte d'Ivoire, Ghana, Guinée Equatoriale, Togo, Zaïre. Document de travail. FAO, Rome, Italie, 43p.

Huguenin J., Blanfort V., Navegantes L., Dufour M., 2010. Configuration of livestock rearing areas in order to maintain the stability of forage systems considering the biophysical hazards of humid tropical climates. Example in French Guyana. Adv. Anim. Biosci., doi:10.1017/S2040470010000592.

IFA, International Fertilizer Industry Association). IFADATA statistical databases. http://www.fertilizer.org/ifa/

IPCC, 2006. IPCC guidelines for national greenhouse gas inventories. Agriculture, forestry and other land use. Vol 4. http:/ /www.ipcc-nggip.iges.or.jp/public/2006gl/ index.html

IPCC 2007a. Climate change 2007: the scientific basis. Contribution of Working Group I to the third assessment report of the Intergovernmental Panel on Climate Change. Cambridge University Press. Cambridge, UK, NY, USA, 996p.

IPCC 2007b. Climate Change 2007: Mitigation. Contribution of Working Group III to the Fourth Assessment Report of the Intergovernmental Panel on Climate Change. Metz B., Davidson O.R., Bosch P.R., Dave R., Meyer L.A. (Eds). Cambridge University Press. Cambridge, UK, NY, USA, 851p.

Kautsky N., Berg H., Folke C., Larsson J., Troell M., 1997. Ecological footprint for assessment of resource use and development limitations in shrimp and tilapia aquaculture. Aquacult. Res., 28, 753-766.

Kaushik S., 2009. Nutrition et alimentation des poissons : éléments pour un nouveau développement. Cah. Agric., 18, 100-102.

Lal R., 2004. Soil carbon sequestration impacts on global climate change and food security. Science, 304, 1623-1627.
Landais E., Lhoste P., 1993. Systèmes d'élevage et transferts de fertilité dans la zone des savanes africaines. Cah. Agric., 2, 9-25.

Lazard J., 2009. La pisciculture des tilapias. Cah. Agric., 18, 174-192

Lipper L., Dutilly-Diane C., McCarthy N. 2010. Supplying carbon sequestration from West African rangelands: opportunities and barriers. Rangeland Ecol. Manage. 63, 155-166.

Li S.F., 2003. Aquaculture research and its relation to development in China. In Agricultural development and the opportunities for aquatic resources research in China Zhang L.X., Liu J., Li S.F., Yang N.S., Gardiner P.R. (Eds). Penang, Malaysia, WorldFish Center, 17-28.

Madec F., Hurnik D., Porphyre V., Cardinale E., 2010. Good practices for biosecurity in the pig sector - Issues and options in developing and transition countries. FAO Anim. Prod. Health Paper, 169, 89p.

Martin C., Morgavi D.P., Doreau M., 2010. Methane mitigation in ruminants: from microbe to the farm scale. Animal, 4, 351-365.

Malhi Y., Roberts T., Betts R.A., Killeen T.J., Li W., Nobre C.A., 2008. Climate change, deforestation, and the fate of the Amazon. Science, 319, 169-172.

Millennium Ecosystem Assessment, MEA 2005. Current state and trends assessment, Washington D.C., Island Press, $815 \mathrm{p}$.

Naylor R.L., Goldberg R.J., Primavera J.H., Kautsky N., Beveridge M.C.M., Clay J., Folke C., Lubchenco J., Mooney H., Troell M., 2000. Effects of aquaculture on world fish supplies. Nature, 405, 1017-1024.

Nguyen, T.L.T., Hermansen J.E., Mogesen L., 2010. Environmental consequences of different beef production systems in the EU. J. Cleaner Prod., 18, 756-766.

Poccard-Chapuis R., Ferreira L., Nahum B. Carvalho S., Tourrand J.F., 2010. Cattle production in the Amazon Rainforest: reasons for the success, challenges for ecological intensification. Advances in Animal Biosciences. doi:10.1017/S2040470010001342.

Porphyre V., Nguyen Q.C., 2006. Pig production development, animal-waste management and environment protection: a case study in Thai Binh province, Northern Vietnam. Porphyre V., Nguyen Q.C. (Eds). PRISE publications, Hanoi, Vietnam, 224p.

Prudencio da Silva V., van der Werf H.F., Spies A., Soares S.R., 2010. Variability in environmental impacts of Brazilian soybean according to crop production and transport scenarios. J. Environ. Manag., 91, 1831-1839.

Rey-Valette H., Clément O., Aubin J., Mathé S., Chia E., Legendre M., Caruso D., Mikolasek O., Blancheton J.P., Slembrouck J., Baruthio A., René F., Levang P., Morissens P., Lazard J., 2008. Guide de co-construction d'indicateurs de développement durable en aquaculture. Cirad, Ifremer, INRA, IRD, Universite Montpellier 1. Diffusion Cirad, Montpellier, France, 144p.

Rutishauser E., Wagner F., Hérault B. Nicolini E.A., Blanc L., 2010. Contrasting aboveground biomass balances in a Neotropical rainforest. J. Veget. Sci., 21, 672-682.

Schiere J.B., Ibrahim M.N.M., Keulen H. van, 2002. The role of livestock for sustainability in mixed farming: criteria and scenario stu- dies under varying resource allocation. Agric. Ecosyst. Environ., 90, 139-153.

Schroeder G.L., 1978. Autotrophic and heterotrophic production of microorganisms in intensively manured fish ponds and related fish yields. Aquaculture, 14, 303-325.

Scoones I., 1995. Living with uncertainty. New directions in pastoral development in Africa. International Institut for Environment and Development, London, Intermediate Technology Publications, 210p.

Soussana J.F., Allard V., Pilegaard K. Ambus C., Campbell C., Ceschia E., CliftonBrown J., Czobel S., Domingues R., Flechard C., Fuhrer J., Hensen A., Horvath L., Jones M., Kasper G., Martin C., Nagy Z., Neftel A., Raschi A., Baronti S., Rees R. M., Skiba U., Stefani P., Manca G., Sutton M., Tuba Z., Valentini R., 2007. Full accounting of the greenhouse gas $\left(\mathrm{CO}_{2}, \mathrm{~N}_{2} \mathrm{O}, \mathrm{CH}_{4}\right)$ budget of nine European grassland sites. Agric. Ecosyst. Env., 121, 121-134.

Soussana J.F., Tallec T., Blanfort V., 2010 Mitigating the greenhouse gas balance of ruminant production systems through carbon sequestration in grasslands. Animal, 4, 334-350.

Steinfeld H., de Haan C., Blackburn H., 1997. Livestock and the environment: issues and options. In: Agriculture and the environment. Perspectives on sustainable rural development. Lutz E. (Ed). The World Bank, Washington, DC, USA, 56p.

Steinfeld H., Gerber P., Wassenaar T., Caste V., Rosales M., de Haan C., 2006. Livestock's Long Shadow: Environmental Issues and Options. Rome, Italie, FAO, 390p.

Steinfeld H., Mooney H.A., Schneider F., Neville L.E., 2010. Livestock in a changing landscape, Volume 1, Drivers, consequences, and responses. Steinfeld H., Mooney H.A., Schneider F., Neville L.E. (Eds). Washington, DC, Island Press, USA, 396p.

Suttie J.M., Reynolds S.G., Batello C., 2005. Grasslands of the world. FAO Plant Production and Protection Series, 34, 538p. ftp:/ /ftp.fao.org/docrep/fao/008/y8344e/y8344e.zip

Thornton C., Shanahan M., Williams J. 2003. From wetlands to wastelands: impacts of shrimp farming. SWS Bull., 48-53.

Thorpe A., 2009. Enteric fermentation and ruminant eructation: the role (and control?) of methane in the climate change debate. Climatic Change, 93, 407-431.

Tilman D., Cassman K.G., Matson P.A. Naylor R., Polasky S., 2002. Agricultural sustainability and intensive production practices. Nature, 418, 671-677.

Tourrand J.F., Piketty M.G., Oliveira J.R.D. Thales M.C., Alves J.B., Veiga J.B., PoccardChapuis R., 2004. Élevage bovin, déforestation et developpement regional : le cas du Sud du Pará Amazonie brésilienne. Bois Forêts Tropiques, 280, 5-16.

Toutain B., De Visscher M.N., Dulieu D., 2004. Pastoralism and Protected Areas: Lessons learned from Western Africa. Human Dimensions of Wildlife, 9, 287-295.

UNFCCC, 2008. Challenges and opportunities for mitigation in the agricultural sector 101p. http://unfecc.int/resource/docs/2008/tp/ 08.pdf.

Veiga J.B., Tourrand J.F, Piketty M.G, Poccard-Chapuis, Alves A.M, Thales M.C., 
2004. Expansão e Trajetórias da Pecuária na Amazônia: Pará, Brasil. Brasília, Editora Universidade de Brasília, Brésil, $162 \mathrm{p}$.

Vigne J.D., 2004. Les débuts de l'élevage. Les origines de la culture. Ed. Le Pommier, Paris, France, 186p.
Vu T.K.V., Tran M.T., Dang T.T.S., 2007. A survey of manure management on pig farms in Northern Vietnam. Livest. Sci., 112, 288-297.

Wassenaar T.P., Gerber P., Verburg P.H., Rosales M., Ibrahim M., Steinfeld H., 2006. Projecting land use changes in the Neotropics: the geography of pasture expansion into forest. Global Env. Change, 17, 86-104.
Wilson E.O., 1989. Threats to biodiversity. Scient. Am., 261, 108-116.

World Bank, 2009. Minding the stock. Bringing public policy to bear on livestock sector development. Report no 44010-GLB, World Bank, Washington, 674p.

\section{Résumé}

Les interactions entre l'élevage et l'environnement sont l'objet d'un débat médiatique, sociétal et scientifique depuis les années 70 . Ce débat, focalisé sur les impacts négatifs de l'élevage sur l'environnement et jusqu'ici limité aux pays développés, concerne dorénavant les pays émergents et en développement où se fera l'essentiel de l'accroissement de la production animale. Toutefois, il est désormais admis que l'élevage peut aussi produire des services écosystémiques en plus de sa fonction productive. Cette synthèse propose un bilan des impacts et des services environnementaux liés aux productions animales dans les pays du Sud et trace des pistes d'amélioration pour les élevages de ruminants, les porcs et volailles ainsi que l'aquaculture. Les changements historiques d'utilisation des terres et de certains habitats aquatiques en cours dans le monde tropical nécessitent une réflexion pour des systèmes de production plus durables. Les émissions de gaz à effet de serre concernent en grande partie les zones tropicales où les systèmes à faible productivité sont forts émetteurs par kg de lait ou de viande produite. Elles sont compensées en partie par un stockage de carbone dans les pâturages, mais les références en zone tropicale restent à préciser. L'augmentation rapide des productions animales au cours des dernières décennies est essentiellement issue de l'industrialisation de l'élevage dans de nombreux pays du Sud où les questions sur la pollution des sols, des eaux, de l'air et le traitement des effluents deviennent incontournables. Les systèmes extensifs moins productifs mais moins polluants conduisent aussi à des services environnementaux. L'élevage est fort consommateur d'eau, mais principalement d'eau «verte» liée à la production végétale. La consommation de ressources fossiles (énergie, phosphates...) s'avère fortement dépendante de l'intensification des systèmes d'élevage. Le risque d'érosion de la biodiversité animale et végétale est une menace avérée, en lien avec la déforestation et les pratiques d'élevage. Dans une discussion globale, les principaux systèmes d'élevage des pays du Sud sont évalués à l'aune de leurs performances environnementales. Des perspectives d'évolution vers des systèmes de production à plus faible impacts et intégrant des services écologiques existent notamment par la mise en place de mécanismes financiers incitatifs. Mais les priorités de lutte contre la faim et la pauvreté constituent des obstacles de taille dans de nombreux pays du sud. Elles conditionnent l'amélioration des performances environnementales à la mise en place de systèmes d'élevage assurant la réponse aux besoins alimentaires des populations et la durabilité économique et sociale indispensable aux sociétés en développement.

\section{Abstract}

\section{Livestock environmental impacts and services in warm regions}

Animal productions are now questioned by the society for their environmental impact. Emerging and developing countries are now concerned by this debate, especially because the increase in animal production in the next decades will take place in these countries, which are often situated in warm regions (including Mediterranean, subtropical and tropical areas). It is now admitted that livestock farming may result in environmental services, besides its productive function. The objective of this paper was to review and discuss environmental impacts and services in southern countries for ruminants, pigs and poultry farms and for aquaculture, and to propose possible solutions for an improvement of environmental performances. Degradation of soil and habitat requires looking for more sustainable production systems. Greenhouse gase emissions are significant in the tropics, especially with low productive systems where the amount per $\mathrm{kg}$ of milk or meat is taken as a reference. This emission is compensated for by carbon sequestration in grasslands; however there is a lack of accurate references in tropical countries. Farming change towards industrialization in many southern countries results in an increase in soil, air and water pollution. Extensive systems are less polluting, and lead sometimes to environmental services. Livestock farming needs water, but this is mainly «green» water related to vegetal production. The use of fossil resources (energy, phosphates, etc.) is highly dependent on the intensification of farming systems. At last, the risk of decrease in animal and vegetal biodiversity is high, in relation with deforestation and farming practices. The main farming systems in southern countries are discussed in relation with their environmental performances. Financial incentives for environmental services may result in low-impact production systems. But the alleviation of poverty and the need for feed security are major concerns which could limit the increase of environmental performances.

BLANFORT V., DOREAU M., HUGUENIN J., LAZARD J., PORPHYRE V., SOUSSANA J.-F., TOUTAIN B., 2011. Impacts et services environnementaux de l'élevage en régions chaudes. In : Numéro spécial, Elevage en régions chaudes. Coulon J.B., Lecomte P., Boval M., Perez J.M. (Eds). INRA Prod. Anim., 24, 89-112. 
